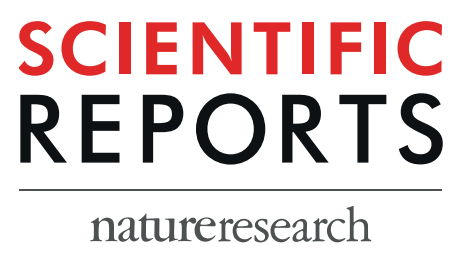

\title{
OPEN Tracking Gold Nanorods' Interaction with Large 3D Pancreatic-Stromal Tumor Spheroids by Multimodal Imaging: Fluorescence, Photoacoustic, and Photothermal Microscopies
}

\author{
Emilie Darrigues $\mathbb{D}^{1 *}{ }^{*}$, Zeid A. Nima ${ }^{1}$, Dmitry A. Nedosekin ${ }^{2}$, Fumiya Watanabe ${ }^{1}$, \\ Karrer M. Alghazali ${ }^{1}$, Vladimir P. Zharov ${ }^{2}$ \& Alexandru S. Biris ${ }^{1 *}$
}

Pancreatic cancer is one of the most complex types of cancers to detect, diagnose, and treat. However, the field of nanomedicine has strong potential to address such challenges. When evaluating the diffusion and penetration of theranostic nanoparticles, the extracellular matrix (ECM) is of crucial importance because it acts as a barrier to the tumor microenvironment. In the present study, the penetration of functionalized, fluorescent gold nanorods into large $(>500 \mu \mathrm{m})$ multicellular 3D tissue spheroids was studied using a multimodal imaging approach. The spheroids were generated by coculturing pancreatic cancer cells and pancreatic stellate cells in multiple ratios to mimic variable tumor-stromal compositions and to investigate nanoparticle penetration. Fluorescence live imaging, photothermal, and photoacoustic analysis were utilized to examine nanoparticle behavior in the spheroids. Uniquely, the nanorods are intrinsically photoacoustic and photothermal, enabling multiimaging detection even when fluorescence tracking is not possible or ideal.

Nanomedicine for cancer treatments-the use of nanosized structures in cancer therapeutics and/or imaginghas been heavily investigated over the last two decades ${ }^{1}$. Nano-sized delivery systems are useful theragnostic agents for enhanced tumor penetration, accumulation, and targeting potency. Studies of nanoparticle (NP)-based therapies have mainly focused on targeted cancer cell treatment but also include cancer stem cells ${ }^{2}$, stromal cancer microenvironment ${ }^{3-5}$, and/or cellular immune system ${ }^{6,7}$. As a physical barrier that limits NP penetration and distribution, the extracellular matrix (ECM) is a crucial concern when evaluating the effects of nanoscale cancer therapies. Recent work showed that the ECM can be regulated by using gold NPs to interrupt the crosstalk between cancer cells and stellate cells, which reeducates the stellate cells ${ }^{8,9}$.

However, the development of nanosystems for cancer treatment has been hampered by the limitations of current in vitro models, which are generally based on two-dimensional (2D) cell cultures. These 2D models struggle to provide an accurate representation of the in vivo environment and its components, which include the dynamic tumor microenvironment (TME), cell heterogeneity, and nutrient and $\mathrm{pH}$ gradient interaction between cells and the $\mathrm{ECM}^{10}$. The real TME is composed not only of cancer cells but also of others cells such as fibroblasts, cancer-associated fibroblasts (CAFs), stromal cells, myofibroblasts, endothelial cells, adipocytes, various immune cells, and extra-abundant compromised ECM. These heterocellular components can induce adaptive survival mechanisms of cancer such as treatment resistance, a leading cause of cancer-related mortality and one of the greatest challenges in cancer treatment ${ }^{11}$. The cellular responses and cell signaling that take place in the TME often cannot be mimicked in a $2 \mathrm{D}$ in vitro model.

${ }^{1}$ Center for Integrative Nanotechnology Sciences, University of Arkansas at Little Rock, 2801 S University Avenue, Little Rock, AR, 72204, USA. ${ }^{2}$ Arkansas Nanomedicine Center, University of Arkansas for Medical Sciences, 4301 West Markham Street, Little Rock, AR, 72205, USA. *email: exdarrigues1@ualr.edu; asbiris@ualr.edu 
The limitations of 2D models have affected the development of nanomedicine approaches to treat pancreatic cancer ${ }^{12}$, particularly pancreatic ductal adenocarcinoma (PDAC) ${ }^{13}$. PDAC is composed of cancer cells, as well as pancreatic stellate cells (PSCs) differentiated into CAFs ${ }^{13}$. These cells, which are similar to myofibroblasts and originate in exocrine areas of the pancreas, are homeostatic, quiescent, lipid-storing cells. When activated by pro-inflammatory cancer signaling, these cells may proliferate, migrate, and accumulate in the tumor by secreting a considerable amount of ECM, which develops a protective, growth-permissive fibrotic stroma barrier. Activated PSCs and CAFs are highly modulated by paracrine/autocrine cell signaling and are involved in the full remodeling of the TME, inducing hypoxia and $\mathrm{pH}$ variation ${ }^{14-17}$. Consequently, CAFs influence PDAC disease progression, tumorigenesis, metastasis, immune reaction, therapeutic response, drug resistance, and treatment failure ${ }^{18-21}$. Multicellular, 3D pancreatic tumor models co-cultured with CAFs have been shown to simulate abundant desmoplasia with ECM. For example, a 3D PDAC in vitro model, made by co-culturing pancreatic cancer cells and stellate cells, generated a rich, dense, active, and mechanically stable stromal compartment ${ }^{22,23}$, relatively similar to that of real tumors seen in biopsied tissues. Based on these results, the $3 \mathrm{D}$ in vitro culture model could be highly useful for PDAC-oriented nanomedicine screening ${ }^{10,24-26}$.

Nanoparticles can have specific light interaction characteristics, making them suitable for multimodal detection, such as Raman spectroscopy ${ }^{27-29}$, photoacoustic analysis ${ }^{30}$, magnetic resonance imaging ${ }^{31}$, two-photon microscopy ${ }^{32}$, or even a combination of these methods ${ }^{33}$, in 2D, 3D and in-vivo models ${ }^{34}$. However, few of these techniques have been applied in $3 \mathrm{D}$ cancer models (spheroids or phantoms) ${ }^{35}$. Imaging-guided NP tracking is a major medical need and research focus, as it would enable drug release to be tracked and mapped in real-time ${ }^{36}$. Recently, a doxorubicin-loaded polymeric NP diffusion was tracked via confocal scanning microscopy in a complex pancreatic cancer 3D model made of cancer, endothelial, and fibroblast cells. Results showed that confocal scanning microscopy is limited in its ability to provide accurate deep penetration $(>100 \mu \mathrm{m})$ data on the diffusion of small molecules due to the progressive loss of their fluorescence signal ${ }^{37}$. This problem may be addressed by utilizing different characterization instruments based on the specific light-matter properties needed.

Additionally, drug-loaded nanocarriers can be tailored to be responsive to stimuli such as temperature, $\mathrm{pH}$, light, magnetic stimulation, and enzymatic processes, which enhances their potential as highly functional drug delivery systems $s^{38-40}$. For example, a series of chemical conjugations (EDC/NHS (1-Ethyl-3(3-dimethylaminopropyl)carbodiimide/ N-hydroxysuccinimide) reaction, protonation/deprotonation balance) was used to cause a $\mathrm{pH}$-responsive bond, enabling $\mathrm{pH}$-triggered controlled drug release from the nanosystem into the tumor tissue $\mathrm{e}^{41,42}$. Because 3D cancer models (especially those composed of stromal cells and ECM components) are designed to mimic the complex in vivo environment with low $\mathrm{pH}$-induced by hypoxic conditionsdrugs can detach off the surface of nanocarriers before accessing cells. Similarly, fluorescent dyes could detach from nanocarriers in certain conditions, which makes tracking based on fluorescence signals less reliable. Despite these possible shortcomings, 3D models are still excellent tools for gaining insight on how multifunctional nanocarriers interact with in vivo systems.

Previously, we successfully used gold nanoparticles' unique photoacoustic (PA) and photothermal (PT) signatures to study their theragnostic behavior and mechanisms of interactions with cells in $2 \mathrm{D}$ culture and in vivo cancer and immune system models ${ }^{43-48}$. Herein, we have developed an avascular 3D pancreatic microtumor model by varying the ratio of Panc-1/PSCs. These spheroids were incubated with gold nanorods (AuNRs, aspect ratio $~ 3$ ), which had been functionalized with a biocompatible thiolated-polyethylene (HS-PEG-COOH) and conjugated with a fluorescent dye (BOPIDY type, $\mathrm{NH}_{2}$-TR-BDP). The first goal of this study was to confirm the ability of our $\mathrm{pH}$-triggered gold nanoparticles to interact with a complex and large $(>500 \mu \mathrm{m}) 3 \mathrm{D}$ pancreatic model. Fluorescence and PA microscopy analyses of the whole spheroids were used to investigate the spheroids' interaction with the NPs and determine the effect of the stroma on NP distribution within the complex spheroid multicellular environement. The second goal of the study was to learn if the data collected for the whole sphere and for the spheroid slices had similar features and, for the first time to the best of our knowledge, to compare fluorescence (from tag) vs. photothermal signatures (unique to the NP) (Fig. 1A). We utilized the unique, intrinsic PA and PT contrast signals of the NPs to identify them in the spheroids without relying only on fluorescent tagging, which enabled multi-imaging analysis and confirmed that tracked fluorescence signals might not always be related to the NP signal.

\section{Results}

Synthesis and characterization of nanoparticle conjugates. Gold nanorods (AuNR, majoritarily with an aspect ratio of length/diameter: $\sim 3$ ) were synthesized using a previously reported seed-mediated $\operatorname{method}^{45,49}$. Performance, toxicity, and interaction of these AuNRs, functionalized with various substances, with cancer cells have already been analyzed in our previous studies ${ }^{47,48}$. In this work, fluorescent nano-agents (nanorod conjugates) were prepared according to the EDC/NHS coupling reaction using both carboxylic (from HS-PEG-COOH) and amine (from BODIPY-TR-NH ${ }_{2}$ ) functional groups in a one-step approach (Fig. 1B) ${ }^{42}$. The morphology and size of the gold nanorods were determined using transmission electron microscopy (TEM) and atomic force microscopy (AFM) (Fig. 2A). TEM images indicated that the majority of the nanorods had a length of $\sim 35.0 \mathrm{~nm}$ and a width of $\sim 12.0 \mathrm{~nm}^{45,46}$. UV-visible spectra analysis confirmed the attachment of $\mathrm{NH}_{2}-\mathrm{TR}-\mathrm{BDP}$ on the AuNR-S-PEG-COOH, forming AUNR-S-PEH-CO-NH-TR-BDP. The nanosystem showed two peaks at $\sim 605 \mathrm{~nm}$ and $\sim 740 \mathrm{~nm}$, corresponding with the maximum wavelength absorption of free $\mathrm{NH}_{2}-\mathrm{TR}-\mathrm{BDP}$ and the second peak of AuNR (the absorption of the longest dimension of the nanostructure), respectively (Fig. 2B).

Zeta potential was used to verify the UV-visible absorption spectra results by measuring the variation of the surface charge/potential during the different steps of conjugation. The values were $\zeta_{\text {AuNRs }}=38 \mathrm{mV} \pm 2 \mathrm{mV}$, $\zeta_{\text {AuNR-S-PEG-COOH }}=-28 \mathrm{mV} \pm 1 \mathrm{mV}$, and $\zeta_{\text {AuNR-S-PEG-CO-NH-TR-BDP }}=25 \mathrm{mV} \pm 2 \mathrm{mV}$, confirming the conjugation of the fluorescent dye to the nanoparticles (Fig. 2C). The AuNRs' positive potential resulted from their dispersion in $\mathrm{CTAB}$, a surfactant used during synthesis to make them shelf-stable in certain concentrations without 
A

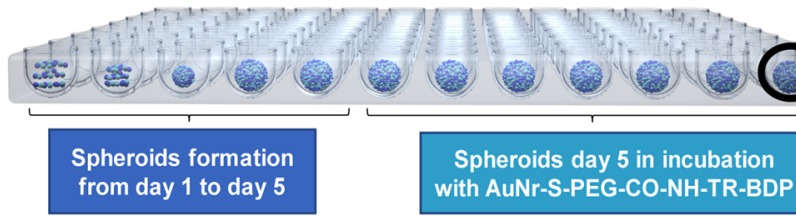

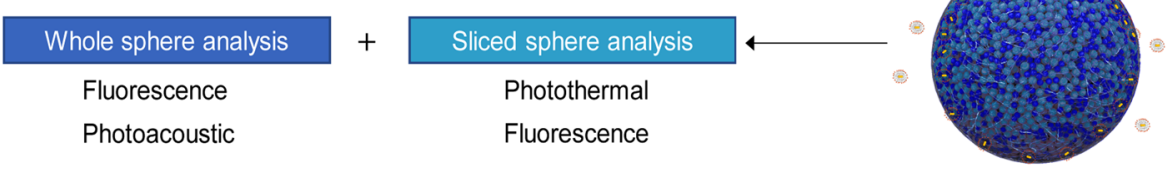

B
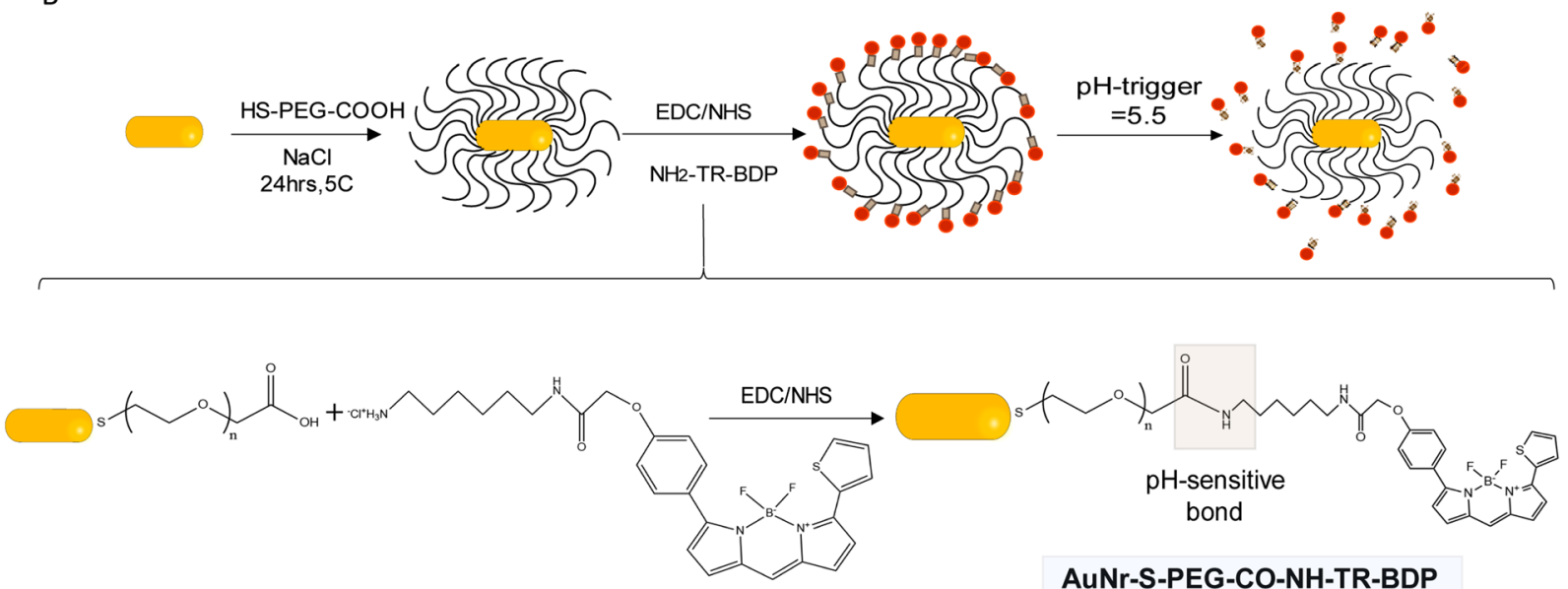

AuNr-S-PEG-CO-NH-TR-BDP

Legend:

Figure 1. Schematic of the whole experimentation and of the AuNRs. (A) Schematic of the experimentation: spheroid formation (day 1 to day 5) in ultra-low-attachment 96-well plate, functionalized AuNR incubation, followed by characterization of the whole sphere and a section of it. (B) Schematic of AuNR functionalization from bare to covered with HS-PEG-COOH to allow EDC/NHS reaction conjugation of the fluorescent dye $\mathrm{NH}_{2}$-TR-BDP, which can be released by acid $\mathrm{pH}$-trigger at a $\mathrm{pH}$ of 5.5 .

sedimentation or structural modification. When the AuNRs were purified and covered by the negatively charged HS-PEG-COOH, their zeta potential became negative, confirming that the conjugation had been successful. However, after the EDC/NHS reaction, the potential of the functionalized fluorescent nanoparticles was raised, becoming positive. Within the structure of $\mathrm{NH}_{2}$-TR-BDP, $\mathrm{NH}_{2}{ }^{+}$is a molecular functional group that can add a positive charge on the surface of the AuNRs. Thus, the nanosystem's positive potential indicates that the NPs were functionalized not exclusively by EDC/NHS covalent bonding but possibly also by other interactions such as electrostatic and/or $\Pi-\Pi$ stacking.

Fluorescence activation release was evaluated by $\mathrm{pH}$ trigger variation at $\mathrm{pH}=7$ using $1 \mathrm{x}$ PBS buffer and at $\mathrm{pH}=5.5 \mathrm{using}$ sodium acetate buffer (Fig. 2Da), with the cells being tracked at $0 \mathrm{hrs}, 0.5 \mathrm{hrs}, 1 \mathrm{hr}, 2 \mathrm{hrs}, 4 \mathrm{hrs}, 6 \mathrm{hrs}$, $24 \mathrm{hrs}, 48 \mathrm{hrs}$, and 5 days. Each point measured the same solution which had previously been dispersed by a sonicator probe in a fresh buffer before incubation at $37^{\circ} \mathrm{C}$. The early sign of release was measured after 1 hour, and the inflection point of the fluorescence release was identified after 6 hours. In the $\mathrm{pH}=7$ solution, after 5 days, a small amount of fluorescent dye had been released (Fig. S1), extremely insignificant compared to $\mathrm{pH}=5.5$, whose release was $\times 10$ times higher for the same time period. At the 5-day point, additional zeta potential measurements of the AuNRs incubated at $\mathrm{pH}=5.5$ were performed. The result for $\zeta_{\text {AuNR-S-PEG-CO-NH-TR-BDP }}(-15 \mathrm{mV} \pm 2 \mathrm{mV})$ (Fig. 2C) also confirmed the release of the dye, with a shift from positive to negative overall charge. 
A

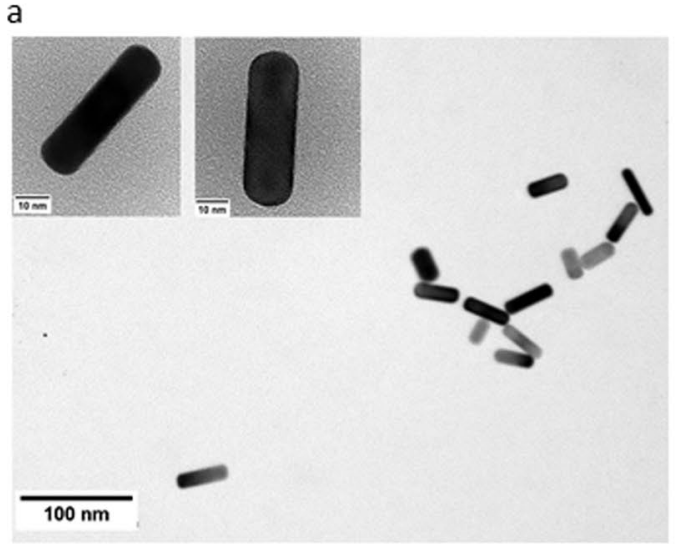

b

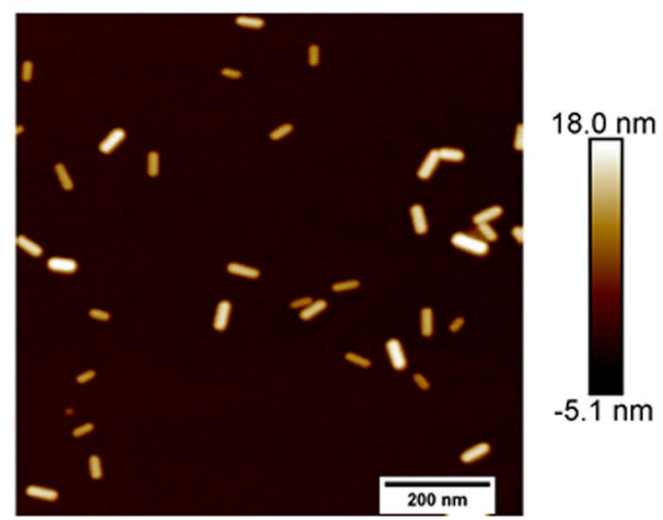

B

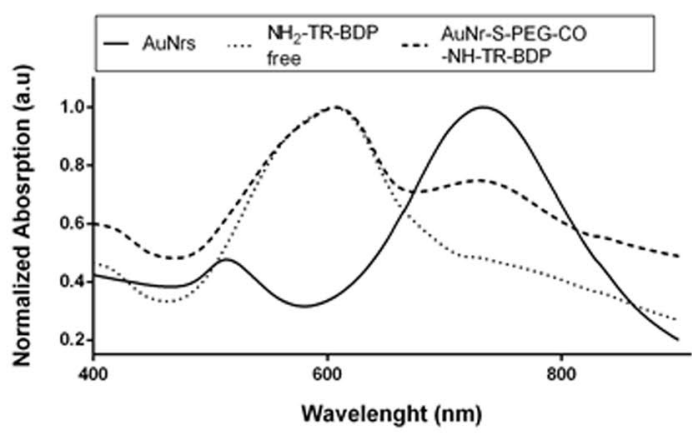

D

a

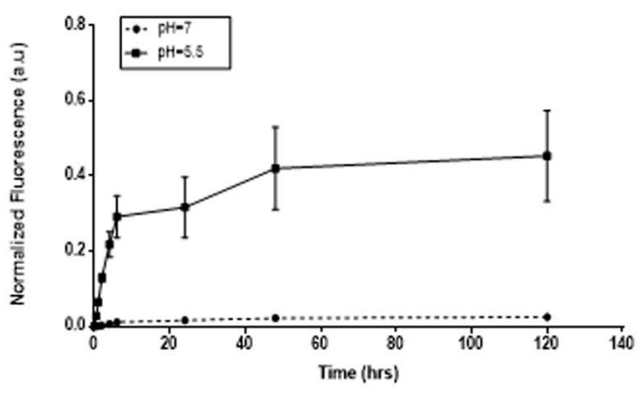

C

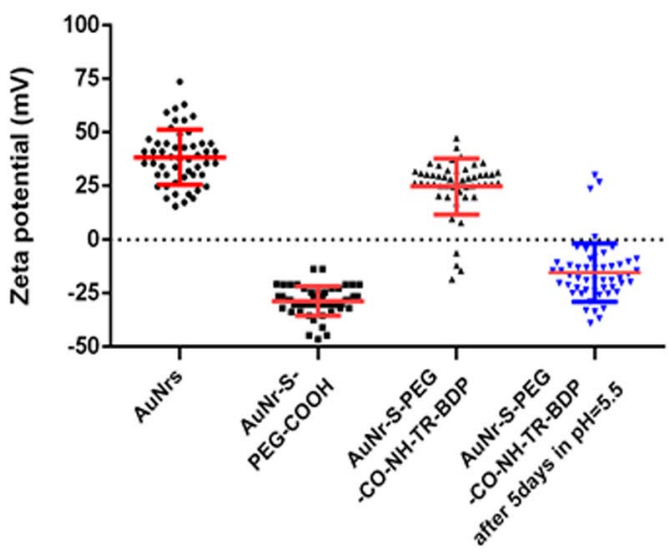

b

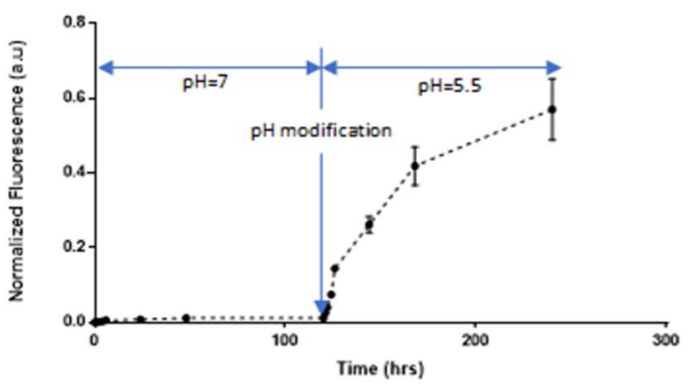

Figure 2. Characterization and analysis of the functionalized AuNRs. (A) (a) High-resolution TEM and (b) AFM images of bare gold nanorods (AuNRs). (B) UV-visible absorption curve of AuNRs, unconjugated $\mathrm{NH}_{2}$-TR-BDP, and fully conjugated AuNR-S-PEG-CO-NH-TR-BDP. (C) Zeta potential measured on AuNRs, AuNR-S-PEG-COOH, AuNR-S-PEG-CO-NH-TR-BDP, and AuNR-S-PEG-CO-NH-TR-BDP after 5 days of exposure to $\mathrm{pH}=5.5$. (D) (a) Normalized fluorescence release intensity of AuNR-S-PEG-CO-NH-TR-BDP incubated at $37^{\circ} \mathrm{C}$ at $5 \% \mathrm{CO}_{2}$ in $\mathrm{pH}=7$ and $\mathrm{pH}=5.5$ for $0.5 \mathrm{hrs}, 1 \mathrm{hr}, 2 \mathrm{hrs}, 4 \mathrm{hrs}, 6 \mathrm{hrs}, 24 \mathrm{hrs}, 48 \mathrm{hrs}$, and 5 days, and, b) Normalized fluorescence release of nanoparticles incubated at $\mathrm{pH}=7$ for 5 days then incubated at $\mathrm{pH}=5.5$ for an additional $30 \mathrm{~min}, 1 \mathrm{hr}, 2 \mathrm{hrs}, 4 \mathrm{hrs}, 6 \mathrm{hrs}, 24 \mathrm{hrs}, 48 \mathrm{hrs}$, and 5 days. This data is a compilation of 3 independent experiments; $n=3$ per group, graph with SEM representation.

Next, the conjugated AuNRs that had been incubated at $\mathrm{pH}=7$ were incubated at $\mathrm{pH}=5.5$ for 5 days. (Fig. 2D,b) These AuNRs produced a fluorescence release after the first minutes of exposure to the acidic buffer. The fluorescence profile confirmed the stability of the conjugated AuNRs at $\mathrm{pH}=7$ and only a possible significant release at $\mathrm{pH}=5.5$, additionally showing that temperature and sonication do not seem to influence the dye release. It was also confirmed that the fluorescence detected in the spheroids could only have originated from the conjugated AuNRs, not from any free dye remaining in the solution or prematurely released before interaction with the cells or spheroid microenvironment. Considering the fast dye release at low $\mathrm{pH}$ values, other biochemical 
reactions/processes (as previously described enzymatic degradation of the disulfide bond PEG-S-S-AuNRs ${ }^{50,51}$ ), the kinetics of fluorescence release inside the cells is expected to occur relatively fast.

Generation and characterization of 3D spheroids. Before investigating the nanoparticles' interaction with the spheroids, we characterized the spheroids. All spheroids were made from cells cultured in 2D on individually cultured Panc-1 (passage $\sim 4$ to 12 ) and PSCs (passage $\sim 3$ to 7 ). The initial total cell seeding number was fixed at $\sim 10,000$ cells/well. The only varied parameter was the co-culture cell ratio of pancreatic cancer to stellate cells, with Panc-1-to-PSCs ratios of 5-1, 1-1, and 1-2. The ratios were determined based on published studies on $3 \mathrm{D}$ pancreatic cancer cells made with stellate cells or various fibroblasts ${ }^{25,52}$. The 1-2 ratio of Panc-1/PSCs was chosen to be in the maximal hypoxic condition.

We performed optical bright field (BF) (Cytation 5) and high resolution scanning electron microscopy (SEM) imaging to monitor the spheroids' growth profile, including real-time size monitoring, optical density analysis, and viability analysis (Fig. 3A-D). Microscopic observation was performed as preliminary data to confirm the sphere-like shape of the spheroids. The sphericity index was investigated by comparing opposite diameter measurements for the same sphere after 5 days. The measurements were relatively similar for all the spheroids, verifying their spherical properties (Panc-1: $0.93 \pm 0.02$, Panc-1-PSC ratio 5-1: $0.92 \pm 0.92$, Panc-1-PSC ratio 1-1: $0.96 \pm 0.01$, Panc-1-PSC ratio 1-2: $0.85 \pm 0.11$ ). SEM images confirmed the spherical structure. Panc-1 only spheroids had a more ellipsoidal structure than the spheroids grown with various ratios of stellate cells because the stellate cells acted as a support or scaffolding to promote a sphere-like shape. SEM analysis provided additional details on the cells' arrangement inside the spheroids but did not allow any visual variations between the different spheroid compositions to be recorded. Additionally, real-time, optical microscopic BF imaging (Figs. 3B and S2A) allowed for daily evaluations of the spheroids' formation parameters as a function of the variations in co-culture ratio.

Spheroid size as a function of incubation time (Fig. 3C) was also evaluated by BF imaging. The results showed different phases of formation: from day 1 to day 3, the cells were sedimented at the bottom of the well and began to organize themselves into spheres. Between days 3-7, the spheroid sizes were stable, but densification was observed, as shown by the optical density evaluation (end point: day 7). After 5 days, the sizes were recorded to be $974 \mu \mathrm{m} \pm 50,952 \mu \mathrm{m} \pm 64,770 \mu \mathrm{m} \pm 49$, and $774 \mu \mathrm{m} \pm 87$ for Panc-1, Panc-1/PSCs ratio 5-1, Panc-1/PSCs ratio 1-1, and Panc-1/PSCs ratio 1-2, respectively. Optical density (Fig. 3D) also indicated a significant variation in the formation and densification processes after day 5, especially for the 1-2 ratio (Panc-1 as a control for the significance study).

Viability testing with calcein AM and propidium iodide (Figs. 3B and S2B) indicated that all the spheres were viable and contained proliferative cells, especially those located in the outer layer (green fluorescence related to the calcein AM). Necrosis (red fluorescence) was mainly observed in the inside of the spheres. However, the necrosis/red fluorescence signal was more dispersed and sporadic in Panc-1 and Panc-1-PSCs ratio 5-1 than in Panc-1-PSCs ratios 1-1 and 1-2, indicating higher necrosis, most likely caused by the higher hypoxic effect that is managed by PSCs and hypothetically resultant from the presence of ECM. Analyzing the size, shape, and morphology of formation revealed the ability of the stellate cells to support the formation of the sphere by "scaffolding" its growth. These results indicate that the model, cell lines, and technique that we developed to obtained 3D cancer model spheroids confirmed observations from previous papers, especially in correlation with the production of a dense and rich $\mathrm{ECM}^{8,22}$.

Nanoparticle interaction observed via fluorescence and PA analysis. Performance, toxicity, and interaction of gold nanorods with various functionalizations with cancer cells have been investigated previously ${ }^{47,48}$. In this study, we used a $50-\mu \mathrm{g} / \mathrm{mL}$ concentration of conjugated nanoparticles for all the spheroid studies because it allows excellent imaging and no toxicity has been detected at that concentration. We focused on two major analyses: fluorescence only (Fig. 4A-C) and photoacoustic signature determination (Fig. 5A-C). Real-time monitoring of fluorescence-conjugated NP incubation was conducted using BioTek Cytation 5. The instrument was set at $37^{\circ} \mathrm{C}$ to maintain normal biological culture conditions. To avoid fluorescence from the background of the media containing dispersed nanoparticles, the instrument was thresholded according to the sample at 20 minutes and according to the control spheroid (spheroid incubated in media without NPs). After 30 minutes, fluorescence was detected in all spheroids, as shown in the $\mathrm{BF}+\mathrm{F}$ (bright field + fluorescence) and $\mathrm{F}$ (fluorescence) images (Fig. 4A,B). Comparison between Panc-1 and Panc-1/PSC ratio 1-2 (Fig. 4B) showed a significant variation in integrated fluorescence after 60 minutes of incubation. This variation was significantly higher in Panc- 1 than in Panc-1/PSC ratio 1-2-reaching 1.2-fold higher after 110 minutes.

Comparison between the stromal-addition spheres in different ratios, with Panc-1/PSC ratio 5-1 as a control, indicated a significant variation of fluorescence after 70 minutes of incubation; ratio 5-1 was 1.2-fold and 1.7-fold higher than ratio 1-1 and ratio 1-2, respectively. Repartition of the fluorescence was also impacted by the ratio of stromal cells. The representative line profile plot of the fluorescence at different times $(30,60,90$, and $110 \mathrm{~min})$ indicated a variation of fluorescence repartition inside the spheres. For Panc-1 and Panc-1/PSC ratio 5-1, the fluorescence increased uniformly in all the spheres as a function of time, but for ratios 1-1 and 1-2, the fluorescence was delayed and remained localized mainly within the outer layers of the spheroids (Fig. 4C). Generally, especially in the case of kinetic fluorescence imaging, spheroid images record non-adherent and floating systems. Consequently, light axial rotation of the sphere can be seen during the measurements, meaning that the images might not look at the same position. However, the measurement of the fluorescence profile was always recorded on the same spheroid position, time point after time point. Based on these results, we concluded that the fluorescence within each sphere is variable, leading us to hypothesize that the concentration and repartition of nanoparticles in each sphere could also be different. These results are in good correlation with published reports, which, by fluorescence tracking of fluorescent-tagged nanoparticles, found that rich stromal spheroids allow limited 
A
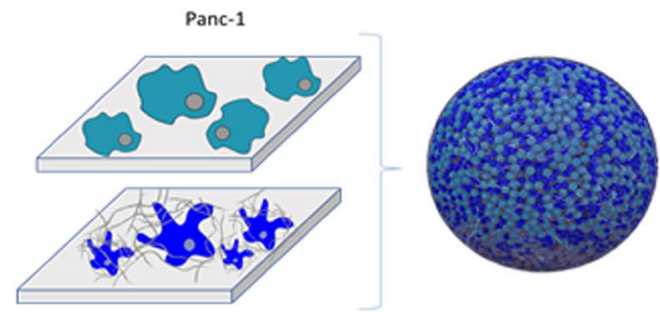

Stellate cells with ECM

B

Panc-1

\section{Bright Field}

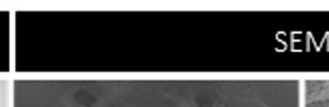

SEM

Calcein AM/PI

Panc-1/ PSCs
Ratio 5-1
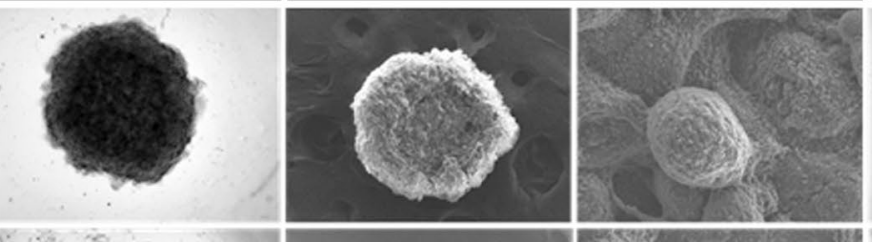

Cantrots
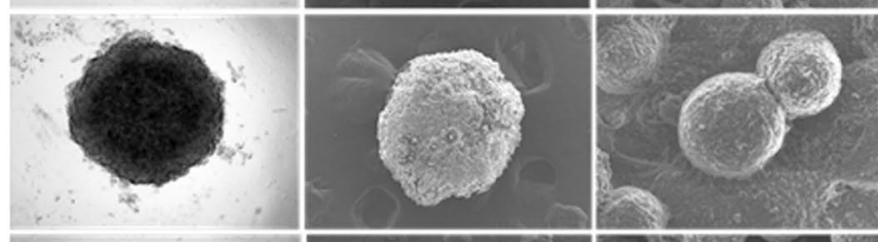

Panc-1/ PSCs

Ratio 1-1
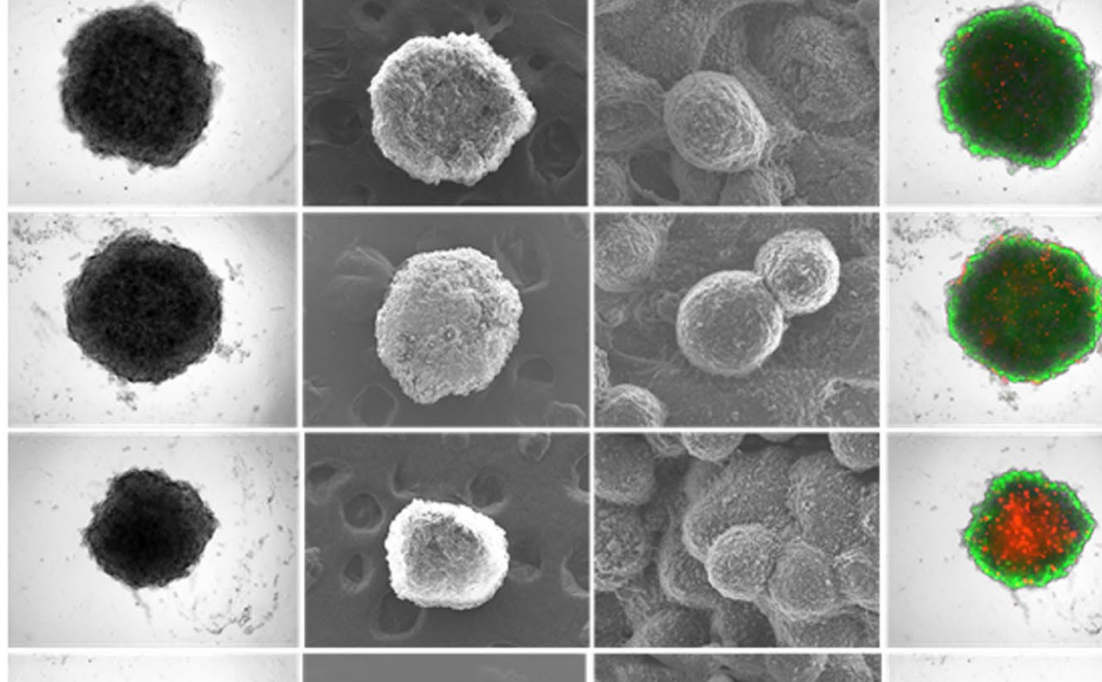

Panc-1/ PSCs

Ratio $1-2$

$\mathrm{C}$
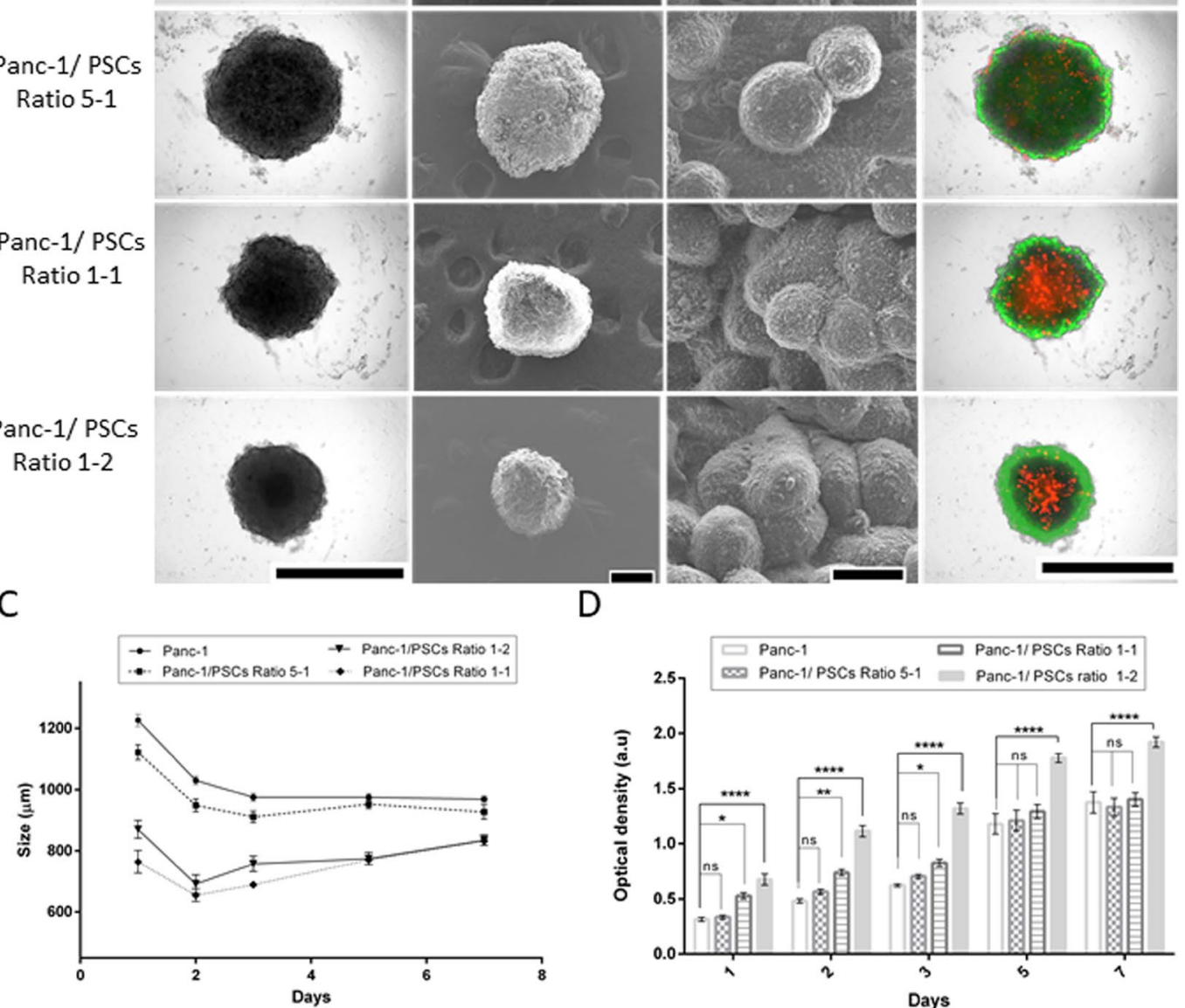

D

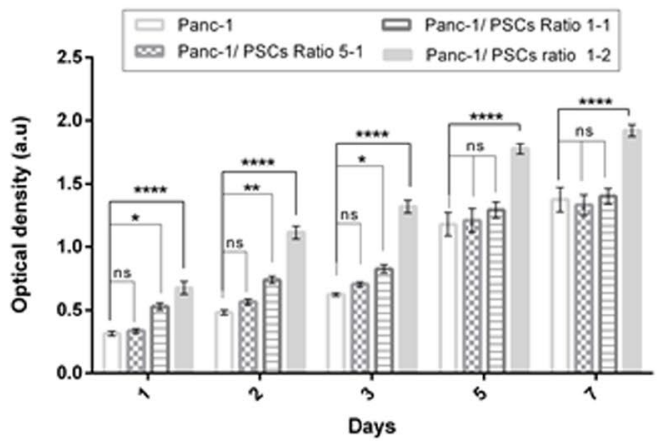

Figure 3. Characterization of the spheroids incubated in a 96-well, ultra-low adhesion plate. (A) Schematic of 3D culture formation from 2D cell culture of Panc-1 and stellate cells. (B) Images of spheroids, from left to right: optical images in the BF (scale bar: $1000 \mu \mathrm{m}$ ), SEM images at magnification x100 (scale bar: $200 \mu \mathrm{m})$ and x3000 (scale bar: $10 \mu \mathrm{m}$ ), and fluorescence optical overlap BF imaging of the proliferation and viability of 3D culture with calcein AM (green)/ propidium iodide (PI, red) (scale bar: $1000 \mu \mathrm{m})$. (C) Evolution of the size and (D) optical density (determined using ImageJ NIH software) of the spheroids at different stellate cell ratios in function of days of formation, from day 1 to day 7 ( $n=20$ in C and D, and $p<0.005$ as determined by two-way ANOVA using Sidak correction in D).

penetration due to the higher amount of ECM components, which hamper and shield the extracellular flow of nanostructures (in an avascular system) $)^{25,38}$.

To verify this hypothesis, we developed a photoacoustic analytic method and performed it on each whole spheroid (Fig. 5). PA data were collected from washed and fixed spheroids previously incubated with $50 \mu \mathrm{g} / \mathrm{mL}$ of 


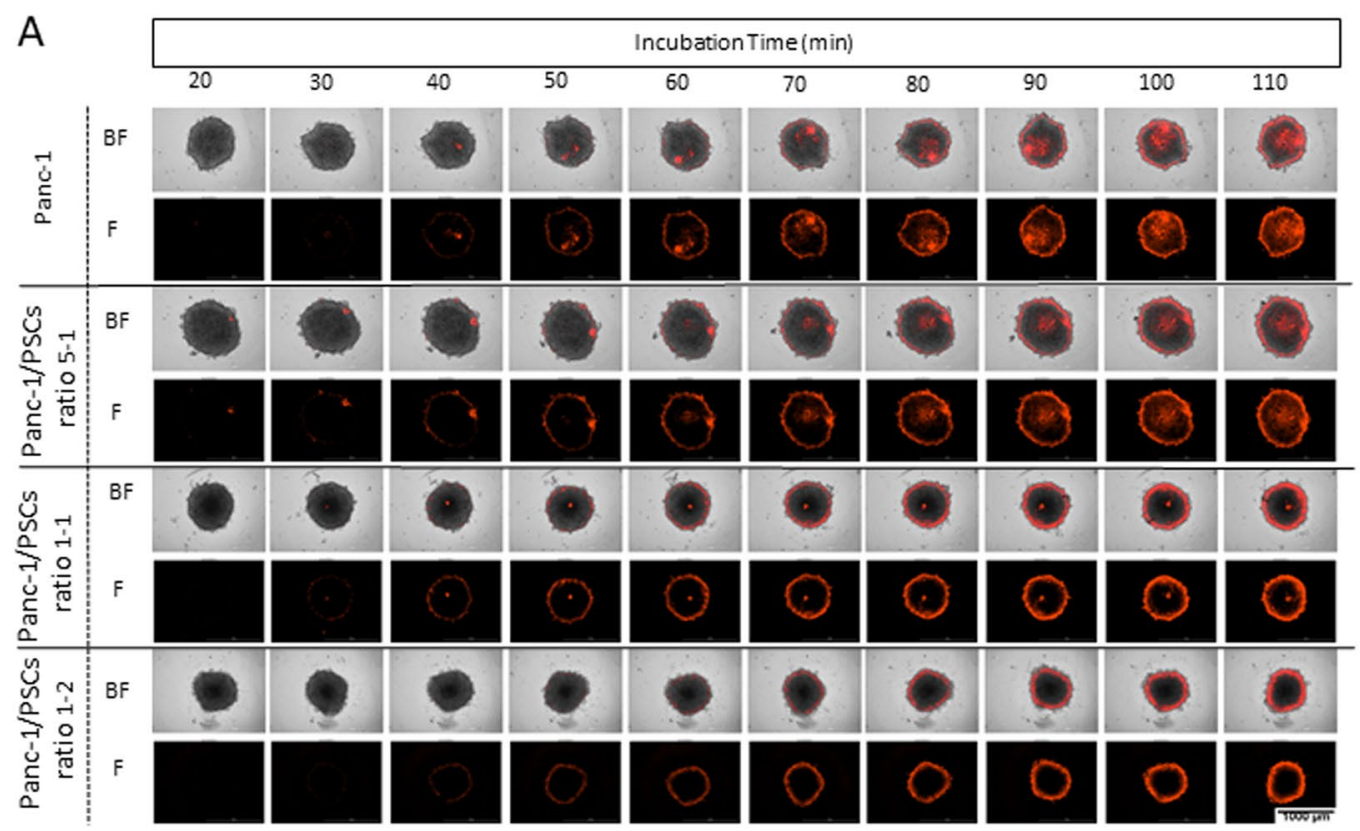

B
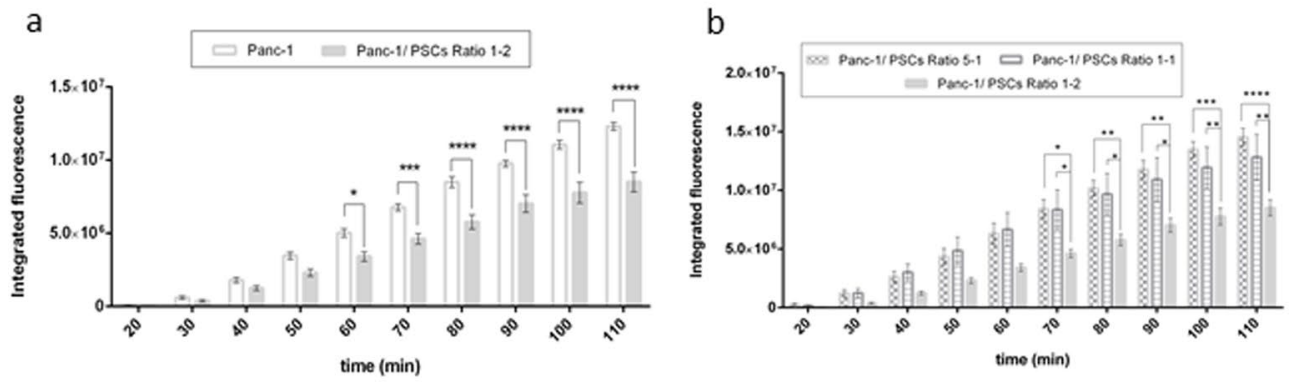

C
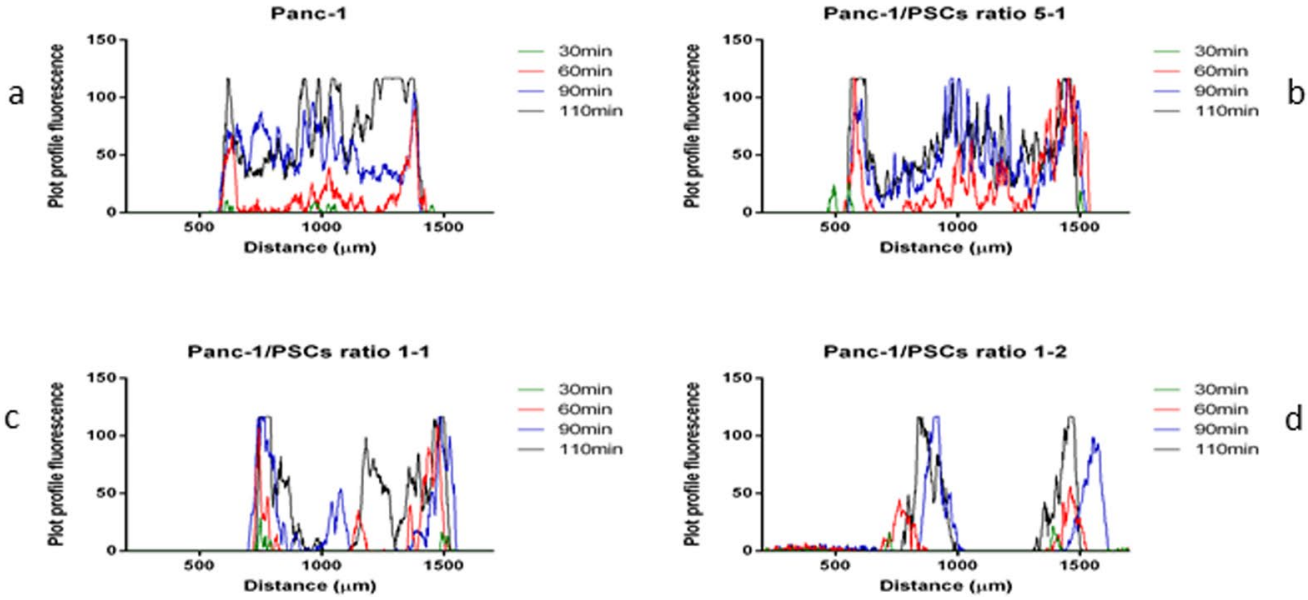

Figure 4. Real-time tracking and kinetic monitoring of the fluorescence on whole spheroids after AuNR-S-PEGCO-NH-TR-BDP incubation. (A) BF and fluorescence (F) images of the different spheroids (Panc-1, Panc-1/ PSC ratio 5-1, Panc-1/PSC ratio 1-1, Panc-1/PSC ratio 1-2) incubated with $50 \mu \mathrm{g} \cdot \mathrm{ml}^{-1}$ of AuNR-S-PEG-CONH-BDP-TR, from $20 \mathrm{~min}$ to $110 \mathrm{~min}$, captured every $10 \mathrm{~min}$. Images recorded with BioTek Cytation 5. (B) (a) Evolution of the integrated fluorescence in Panc-1 and Panc-1/PSC ratio 1-2 in function of incubation time, with significance on the mean of the standard error with Panc- 1 as a control; $\mathrm{p} \leq 0.05$, determined by two-way ANOVA using Sidak correction $(\mathrm{n}=4)$. (b) Evolution of the integrated fluorescence in Panc-1/PSC ratios 5-1, 1-1, and 1-2 in function of incubation time, with significance on the mean of the standard error, with Panc-1/PSC ratio 5-1 as a control, with $\mathrm{p} \leq 0.05$, determined by two-way ANOVA using Sidak correction $(\mathrm{n}=4)$. $(\mathrm{C})$ Representative linear surface plotting based on the mean grey fluorescence plot profile at different times: 30, 60, 90, and 110 min with (a) Panc-1, (b) Panc-1/PSC ratio 5-1, (c) Panc-1/PSC ratio 1-1, and (d) Panc-1/PSC ratio 1-2. 
A

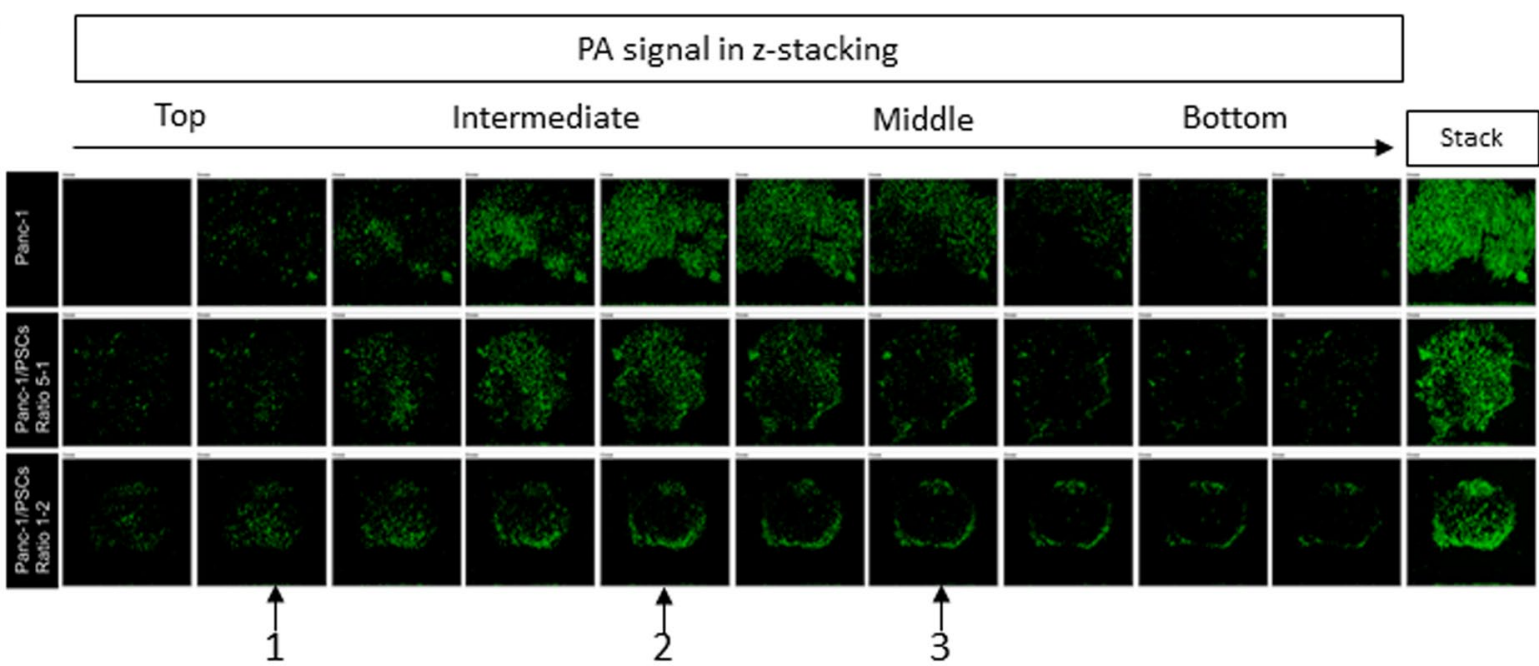

B

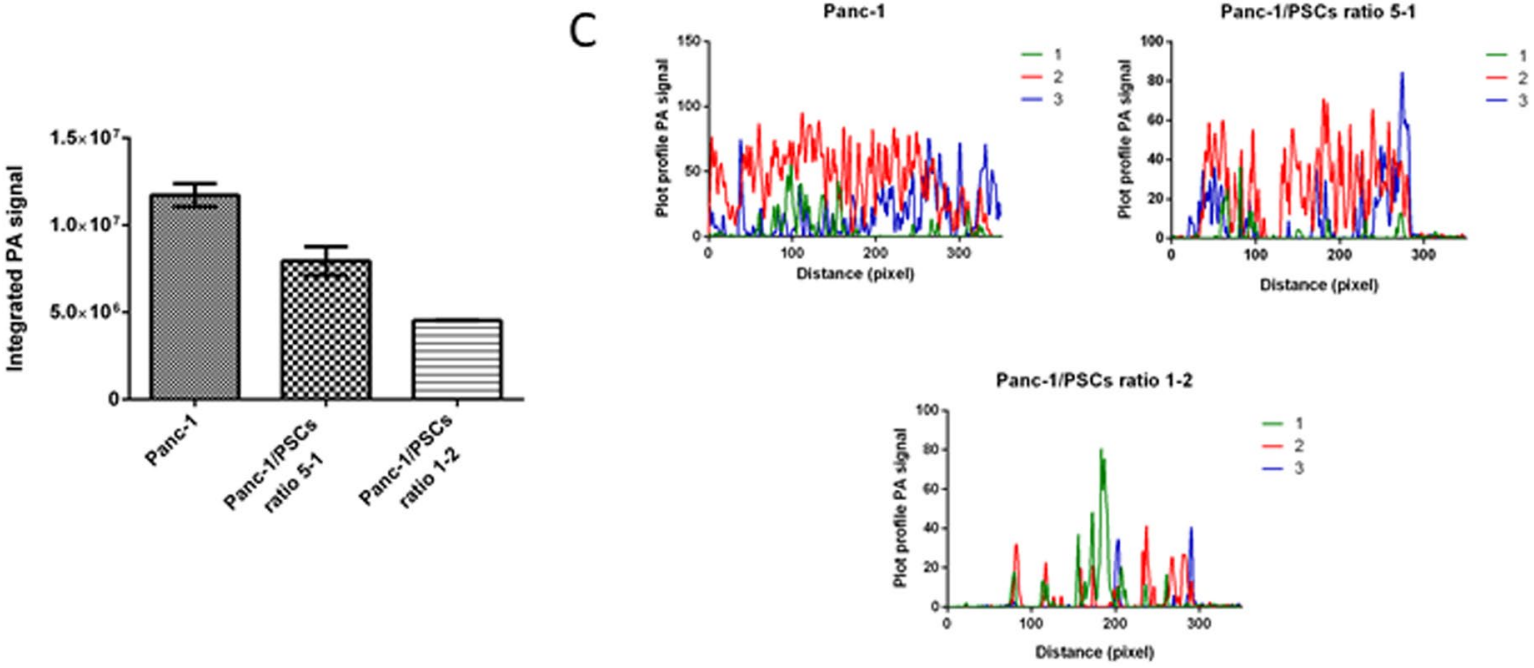

Figure 5. PA imaging on the whole spheroid, recorded by virtual z-stacking after incubation with AuNR-SPEG-CO-NH-TR-BDP for 2 hours. (A) PA imaging in virtual $z$-stack $(z=10 \mu \mathrm{m})$ of the different spheroids (Panc-1, Panc-1/PSC ratio 5-1, Panc-1/PSC ratio 1-2) incubated with $50 \mu \mathrm{g} \cdot \mathrm{ml}^{-1}$ of AuNR-PEG-CO-NHBDP-TR for 2 hours. The arrows numbered 1,2, and 3 at the bottom of the figure indicate the stack pictures used in C to plot the PA signal profile. (B) Evolution of the AuNR-PA signal accumulation recorded in each sphere $(\mathrm{n}=2)$. (C) Line profile of PA signal through stack images 1, 2, and 3 for Panc-1, Panc-1/PSC ratio 5-1, and Panc-1/PSC ratio 1-2.

conjugated AuNRs, immersed in DI water in a petri dish. Each condition was tested twice. Z-stack depth imaging ranging from $10-20 \mu \mathrm{m}$ from the top to the bottom of the spheroids was performed and the images analyzed. The results were in good agreement with the previous fluorescence data. A stack-by-stack comparison of images within the same spheroid also showed variation in the repartition of the PA signal: Panc-1 and Panc-1/PSC ratio 5-1 possessed higher distribution, even in deeper depths inside the spheroids, while the Panc-1/PSC ratio 1-2's signal was mainly concentrated in the outer layers (Fig. 5C). These results are similar to those recorded for the fluorescence plot profiles.

Therefore, a combinatorial approach of Cytation 5 and PA microscopy on the whole spheres allowed us to rapidly (instant imaging) and accurately visualize the distribution of the nanoparticles and the fluorescence of the corresponding dye used as a release model. We observed the ability of the nanoparticles to release the fluorescent dye in acidic conditions, and the PA technique determined the distribution of the nanoparticles within the spheroids based on the unique PA signature of the nanorods.

Slicing of the spheroids and combined fluorescence-PTM analysis. In order to confirm the details of nanoparticle distribution within the spheroid, we utilized high-resolution photothermal microscopy (PTM) that is similar to the PA method utilized to observe the absorption contrast of the NPs but has much higher sensitivity and resolution. Indeed, data recorded in previous studies ${ }^{53,54}$ indicated that PTM offers absorption sensitivity $\geq 30-50$ - fold than PA in vitro and does not require an acoustic contact between the sample and transducer. Additionally, compared to the PA microscope, the PTM was built with a fluorescence microscope addition, 


\section{Whole section fluorescence}

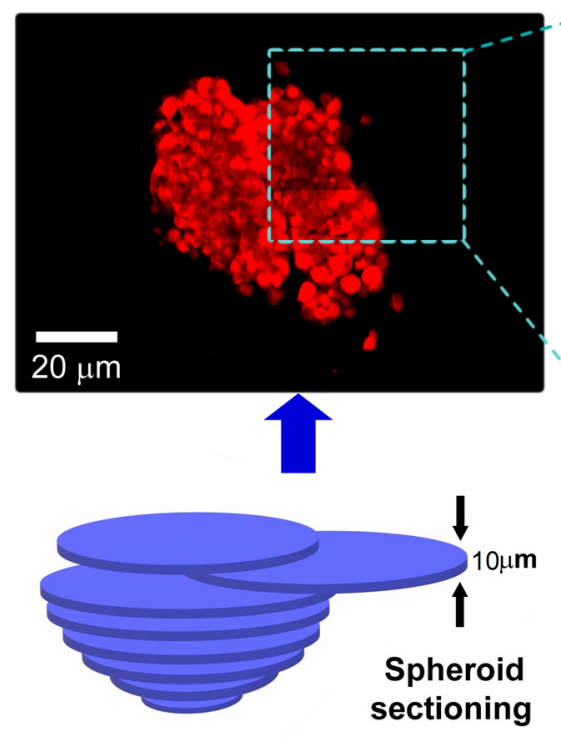

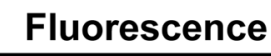
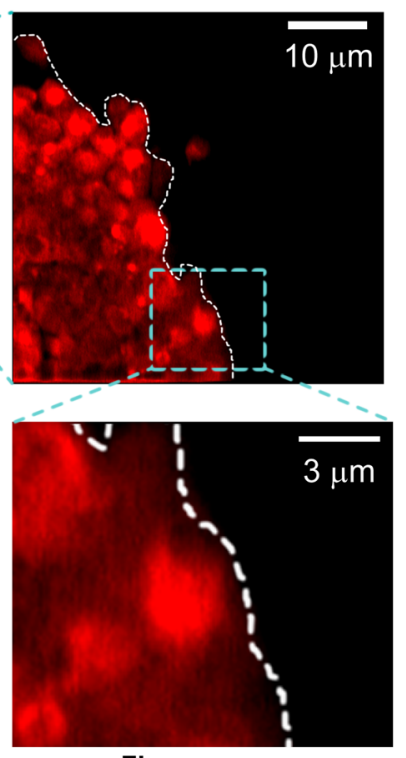

Fluorescence

$\min \max$

\section{Single frame data}

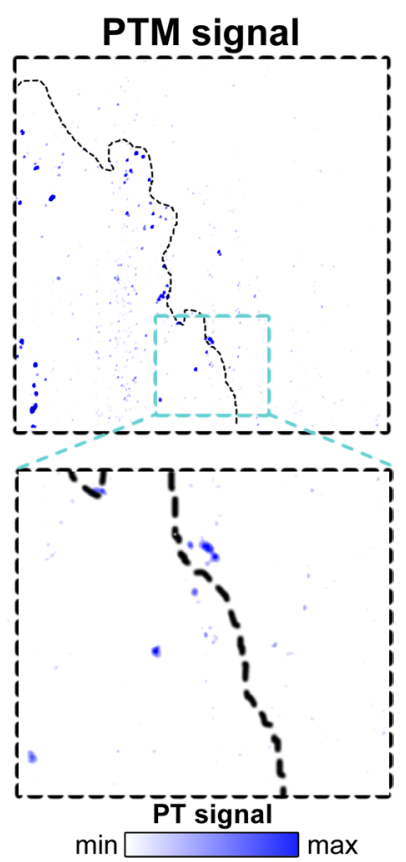

Figure 6. Principles of fluorescence and PTM analysis of spheroid sections. Blue dash line indicates the correlation between zoomed sections and the whole slice. White and black dash lines highlight the edge of tissues on the section.

providing the perfect approach to compare the signals of both techniques. However, our PTM can scan only $\sim 47 \times 47 \mu \mathrm{m}$ squares and requires stitching to recompose the images, which takes a longer time than PA data recording on a large sample (entire spheres). Advanced care needs to be given to the process of stitching in order to make sure that the data is accurately plotted.

In identical conditions as used in the previous experiments, spheroids grown for 5 days were incubated for 2 and 24 hours with $50 \mu \mathrm{g} / \mathrm{mL}$ of conjugated AuNRs. At both time points, they were washed and fixed with paraformaldehyde. They were then sliced at $10 \mu \mathrm{m}$ thickness. Instrumental microscopy, along with photothermal and confocal fluorescence detection, was used on selected slices to accurately locate the top, intermediate, and middle of each spheroid. Figure 6 shows the general principles of spheroid slicing, fluorescence/PTM quantification of the released dye (fluorescence), and NP location (PTM signal) (Fig. 6).

After 2 hours of incubation, fluorescence analysis on the slices showed that in Panc-1, Panc-1/PSC ratio 1-5, and Panc-1/PSC ratio 1-2 spheres, the nanosystems did not seem to have reached the core of the spheroids. The fluorescence analysis of the percentage of the area covered by the fluorescent dye inside the different stacks indicated more than $90 \%$ coverage for all the spheres but dropped to $74 \%$ at $10-\mu \mathrm{m}$ depth for Panc-1/PSC ratio $1-2$. In intermediate and middle slices for all spheres, fluorescence covering dropped below $40 \%$.

Extremely variable results were recorded after 24 hours of incubation: the fluorescent NP coverage of the intermediate and middle slices was $\sim 97 \%, \sim 65 \%$, and 30\% for Panc-1, Panc-1/PSC ratio 5-1, and Panc-1/PSC ratio 1-2, respectively. Between 2 and 24 hours, the fluorescence in the core of Panc-1/PSC ratio 1-2 was unchanged. Also, compared to the analysis performed previously on the whole sphere, a small variation was visible between Panc- 1 and Panc-1/PSC ratio 5-1, with 30\% variation of cover in the deeper layer. PTM images confirmed the presence of NPs and indicated a variation of repartitions in function with the stroma present in the spheroids. Surprisingly, the relative number of NPs, especially within the outer layers of the spheroids, did not show any higher variation between 2 and 24 hours. The only significant variation between 2 and 24 hours was the ability of nanoparticles to flow deeper inside the spheroids, with the following trend: Panc-1 > Panc-1/PSC ratio 5-1 > Panc-1/PSC ratio 1-2. (Figs. 7, S3, and S4). However, it must be pointed out that the fluorescent signals and the NP signals did not always overlap (Fig. S5-video), especially after 2 hours of incubation. Our observations showed that the nanoparticles are processed by the cells, and the fluorescence dye could be released inside the spheroid environment.

As a general remark, we made sure that all the spheroids maintained their integrity during formation and NP incubation. If cracks or surface irregularity are visible, they are from the successive washing and handling, which also indicates that some spheroids, especially Panc-1, are more brittle than those containing stromal cells.

\section{Discussion}

We have successfully developed an avascular, complex, co-culture 3D spheroid model made of human pancreatic stromal cells of different sizes and densities that can be used to study the interaction, penetration, and diffusion of loaded gold nanoparticles. We have demonstrated that these AuNRs enable a multiple-source tracking/ imaging system-fluorescence, photoacoustic, and photothermal microscopy. Through these tracking/imaging methods, we confirmed that stromal cells in this 3D spheroid model act as a physical and/or chemical barrier for 
A
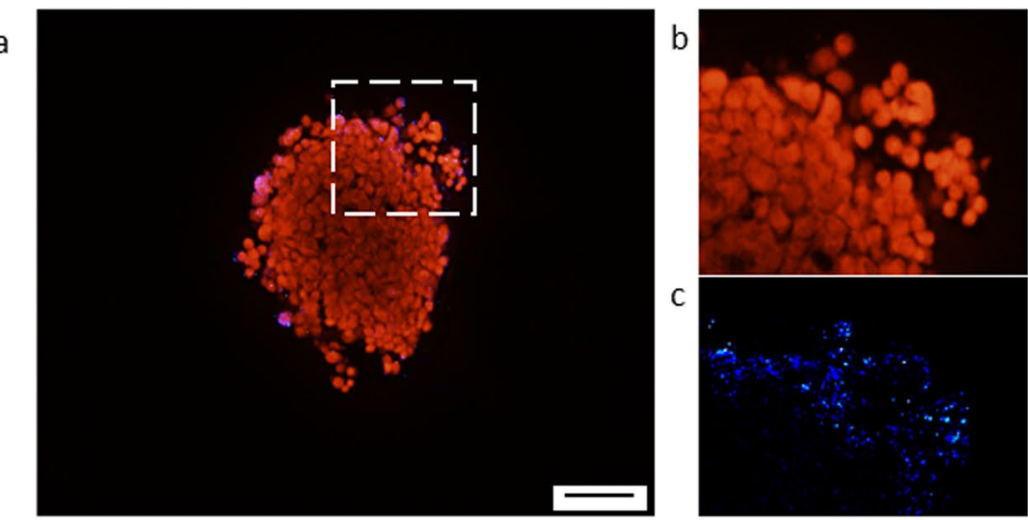

B

\begin{tabular}{ccccccc}
\multicolumn{1}{c}{ Top } & \multicolumn{3}{c}{ Intermediate } & \multicolumn{2}{c}{ Middle } \\
\hline 1 & 2 & 3 & 4 & 5 & 6
\end{tabular}

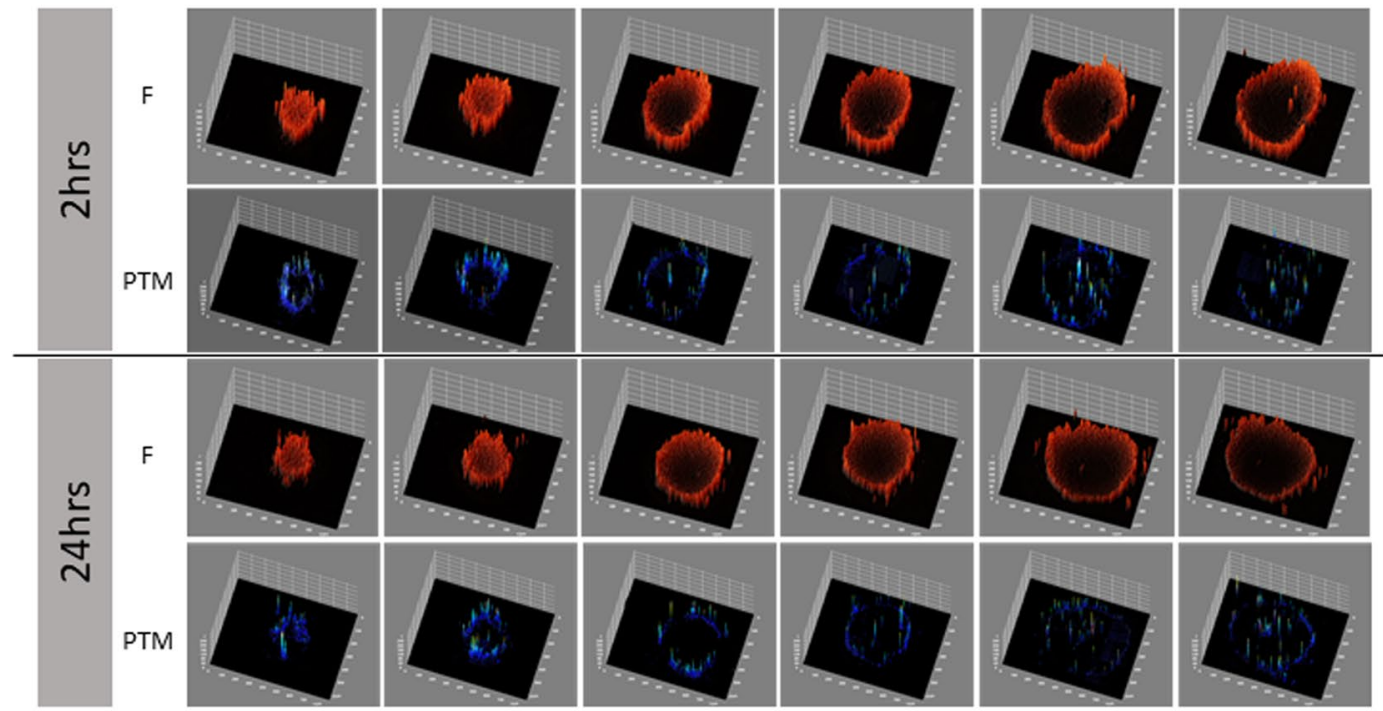

$\mathrm{C}$

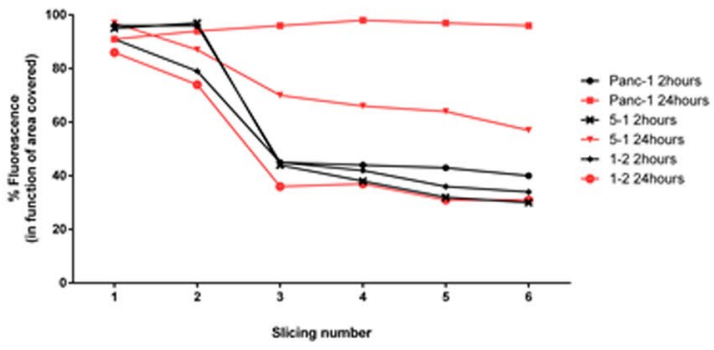

Figure 7. Fluorescence and PTM analysis on sections of the different spheroids after 2 and 24 hours of incubation with $50 \mu \mathrm{g} \cdot \mathrm{ml}^{-1}$ of AuNR-S-PEG-CO-NH-BDP-TR: (A) Fluorescence (red) and PTM (blue) image recorded for slice 1 (representing the top of the spheroid), after 2-hr NP incubation, with (a) PTM and fluorescence images overlapping on the whole slice (scale: $100 \mu \mathrm{m}$ ), (b) fluorescence image of the white rectangle section from (a-c) PTM image of the white section from (a); (B) 3D representations of the fluorescence (F) and PTM images of two slices on the top, intermediate, and middle of Panc-1/PSC ratio 1-2 after incubation. (C) Evolution of percentage of fluorescence (as \% of area covered) in each section in function of incubation time.

gold nanoparticle penetration. We analyzed the massive spheroids (dimension $>500 \mu \mathrm{m}$ ) using a functionalized tracker and the inherent properties of the nanostructure. PTM and PA analysis highlighted the low penetration of the nanoparticles inside the 3D spheroid system.

It is important to note that the AuNRs were found to agglomerate on the outer layer of the 3D spheroids, as identified by their massive PA and PTM signals; these results were influenced by the spheroids' stromal cell 
content. Our hypothesis is that the first layers of cells were quickly saturated, decreasing their ability to uptake more nanoparticles. At the same time, increasing incubation time allowed nanoparticles to penetrate closer to the core, especially if the stroma was not present. Recent studies suggest that endocytosis and interstitial cellular gaps regulate nanoparticle transportation within pancreatic $3 \mathrm{D}$ cancer models ${ }^{52}$.

Interestingly, the fluorescent and the nanoparticle (PTM images) signals did not always overlap (Fig. S5-video), even as early as after 2 hours of incubation. We observed that the NPs are processed by the spheroids, and the fluorescent dye could be released inside the corresponding environment. We also proved that fluorescence tracking to determine NP penetration is dependant of the chemical conjugation between the dye and the nanoparticles; for example, the EDC/NHS reaction and its ultra-labile amide bonding can enable a $\mathrm{pH}$-triggered release.

Analysis and tracking of both the nanoparticles and the fluorescence are necessary in order to accurately determine the efficiency of the release model. In Panc-1 model, due to morphological specificity, both fluorescence and nanoparticles can flow closer to the core, which was not observed for the Panc-1/PSC ratio 1-2 system, possibly due to more corresponding necrosis and ECM, reducing the interstitial space. In consequence, the fluorescent dye-and, by extrapolation, a nanosystem — can have more difficulty being delivered.

This study indicates the need for further investigation of active targeting nanostructures, not only for cancer cells, but also for cancer stroma, and their action on its non-cellular components such as ECM (regulation, degradation), to overcome past failed therapies ${ }^{5,19,55}$.

\section{Methods}

AuNR synthesis and conjugation with $\mathrm{NH}_{2}$-TR-BDP fluorescent dye by EDC/NHS reaction. AuNRs were prepared using previous reported protocols ${ }^{45,47,49}$. First, AuNRs ( 1 batch of $\sim 1,000 \mu g$ ) were prepared from a seed solution. Briefly, the seed solution was prepared by stirring $5 \mathrm{ml}$ of CTAB solution $(0.2 \mathrm{M})$ with $5 \mathrm{ml}$ of $\mathrm{HAuCl}_{4}(0.0005 \mathrm{M})$ and $600 \mu \mathrm{l}$ of $\mathrm{NaBH}_{4}(0.01 \mathrm{M})$. Then, to synthesize AuNRs with an aspect ratio of $\sim 3,5 \mathrm{ml}$ of CTAB $(0.2 \mathrm{M}), 150 \mu \mathrm{l}$ of silver nitrate aqueous solution $(0.004 \mathrm{M})$, and $5 \mathrm{ml}$ of $\mathrm{HAuCl}_{4}(0.001 \mathrm{M})$ were combined. Next, $70 \mu \mathrm{l}$ of ascorbic acid $(0.0788 \mathrm{M})$ and $12 \mu \mathrm{l}$ of seed solution were added, and the solution was kept at $30^{\circ} \mathrm{C}$ for 40 minutes. The AuNRs were then purified twice at 10,000 rpm for 20 minutes and re-dispersed in DI (deionized) water. Second, AuNRs were covered with an HS-PEG-COOH solution. Briefly, the gold nanorods were dispersed in a 2-ml solution of carboxyl thiolated polyethylene glycol (HS-PEG-COOH) $\left(\mathrm{M}_{\mathrm{W}} \sim 3,400\right)$, prepared by dissolving $2 \mathrm{mg} / \mathrm{mL}$ of PEG powder in $2 \mathrm{mM}$ of $\mathrm{NaCl}$ solution, and maintained at $5^{\circ} \mathrm{C}$ for at least 12 hours. The AuNR-S-PEG-COOH were then purified twice at $10,000 \mathrm{rpm}$ for 20 minutes. Finally, the AuNR-S-PEG-COOH were dispersed in $1 \mathrm{~mL}$ of DI water for storing or in $1 \mathrm{xPBS}$ for immediate conjugation. An EDC/NHS reaction ${ }^{42}$ was used to conjugate the functionalized PEG-coated nanorods with the fluorescent dye $\mathrm{NH}_{2}-\mathrm{TR}-\mathrm{BDP}$. $1 \mathrm{~mL}$ of $1000-\mu \mathrm{g} / \mathrm{ml}$ AuNR-S-PEG-COOH was dispersed in $1 \mathrm{x}$ PBS, then $57 \mu \mathrm{L}$ of $10-\mathrm{mg} / \mathrm{mL}$ EDC were added, which had been previously dissolved in 1x PBS. The solution was orbitally shaken for 10-15 minutes. Then, $28 \mu \mathrm{L}$ of $10-\mathrm{mg} / \mathrm{mL}$ NHS in $1 x$ PBS were added, and the solution was shaken again for 10 minutes. $400 \mu \mathrm{L}_{\text {of }} \mathrm{NH}_{2}-\mathrm{TR}-\mathrm{BDP}$, previously dissolved at $100 \mu \mathrm{g}$ in $400 \mu \mathrm{L}$, were added and orbitally shaken for 4 hours. The solution was centrifuged at 10,000 rpm for 20 minutes, washed 3 times, and re-dispersed in $1 \mathrm{~mL}$ of 1x PBS.

$\mathrm{pH}$-controlled fluorescence release. 1 batch of functionalized AuNR-S-PEG-CO-NH-BDP-TR was incubated with $1 \mathrm{ml}$ of $1 \mathrm{x}$ PBS buffer at $\mathrm{pH}=7$ as a control, and 1 batch was incubated with $1 \mathrm{~mL}$ of sodium acetate buffer at $\mathrm{pH}=5.5$. The AuNR-S-PEG-CO-NH-BDP-TR $(\mathrm{pH}=7)$ and AuNR-S-PEG-CO-NH-BDP-TR $(\mathrm{pH}=5.5)$ were sonicated with a sonicator probe for 15 seconds and incubated in the cell incubator/biological culture at $37^{\circ} \mathrm{C}, 5 \% \mathrm{CO}_{2}$. Next, the tubes were centrifuged at $10,000 \mathrm{rpm}$ for 30 minutes, and $100 \mu \mathrm{L}$ of supernatant was collected and introduced in a 96-well-plate. Fresh buffer was introduced in the tube, sonicated for 15 seconds by sonicator probe, then incubated as mentioned before. Fluorescent reading was done with the microplate reader in fluorescence mode (Synergy H1 from BioTek, Vinooshi, VT). The samples were triplicate $(\mathrm{n}=3)$. After 5 days, the samples with a $\mathrm{pH}$ of 7 were centrifuged, and sodium acetate buffer $(\mathrm{pH}=5.5)$ was added. The same protocol as before was used to control the fluorescence release.

Cell culture, 3D formation, and characterization. Panc-1 cells were grown in T75 culture flasks with DMEM containing $10 \%$ fetal bovine serum and $1 \%$ penicillin/streptomycin. Human primary PSCs in T75-poly-L-lysine modified culture flasks were cultured in the medium provided by the manufacturer $\left(\right.$ Sciencell $\left.{ }^{\mathrm{TM}}\right)$. All the cells were maintained in an incubator at $37^{\circ} \mathrm{C}$ in a $5 \% \mathrm{CO}_{2}$ atmosphere and then sub-cultured by trypsinization every 3-5 days, in limited passages ( 8 for PSCs and 14 for Panc- 1 ). The cell culture media used for the 3D culture was DMEM/F12 complemented with $10 \%$ fetal bovine serum and 1\% penicillin/ streptomycin. The cells were initially seeded at $\sim 10,000$ cells in a 96-well, ultra-low attachment plate. Cells were co-cultured at different ratios of Panc-1 to PSCs (1:1, 1:2, and 5:1). Spheroids of Panc-1 alone and/or PSCs alone were used as controls. Media was changed every 2 days. Spheroids were imaged with Cytation 5 from Biotech from day 1 to day 7. BF images were taken using a x10 objective lens. Optical density was evaluated using ImageJ software after correct calibration every day. For viability imaging, the spheroids were grown for 5 days, then the media was removed, and the spheroids were washed at least 2 times with $1 \mathrm{x}$ PBS, previously warmed at $37^{\circ} \mathrm{C}$. Then, $100 \mu \mathrm{L}$ of a solution containing $1 \mu \mathrm{L}$ of calcein $\mathrm{AM}, 1 \mu \mathrm{L}$ of diluted PI, and $98 \mu \mathrm{l}$ of PBS were added for each spheroid. The spheroids were incubated at $37^{\circ} \mathrm{C}$ then imaged with Cytation 5 in fluorescence microscopy mode, using GFP (green fluorescence protein) and TR (Texas red) filters for fluorescence imaging. For SEM imaging, the spheroids were cultured for 5 days in ultra-low adhesion plates. They were washed twice with $1 x$ PBS. Individual spheroids were then immersed in 3\% glutaraldehyde in the $0.1-\mathrm{M}$ cacodylate buffer at $\mathrm{pH} 7.2$ and maintained at $4{ }^{\circ} \mathrm{C}$ for 24 hours maximum. After being washed twice with the cacodylate buffer and twice with DI water, the individual spheroids were immersed in a $2 \%$ buffered solution of osmium tetroxide and maintained at $4{ }^{\circ} \mathrm{C}$ for 24 hours maximum. The spheres were then dehydrated by a series of absolute ethanol washings. Finally, the 
ethanol was removed, replaced by HMDS solution, and left under the hood until complete evaporation. The spheroids were then analyzed using SEM.

Nanoparticle interaction on the whole sphere. The fluorescence analysis of the whole spheroids was conducted on 5 -day-grown spheroids. $50 \mu \mathrm{g} / \mathrm{mL}$ of the nanorod conjugate) was added to each sphere. The 96-well-plate was then placed inside the Cytation5, already maintained at $37^{\circ} \mathrm{C}$, and every 10 minutes, a BF image and a fluorescence image were recorded using an x10 objective lens. Tracking was conducted for 110 minutes. For PA imaging on the whole sphere at 2 hours and 24 hours, the spheres were grown for 5 days, and $50 \mu \mathrm{g} / \mathrm{mL}$ of nanorod conjugate were added to each sphere. At the determined times, the spheroids were washed twice with $1 \mathrm{x}$ PBS and fixed with $4 \%$ PFA for $\sim 24$ hours at $37^{\circ} \mathrm{C}$. A custom laser scanning PA microscope was coupled to an inverted Olympus IX81 microscope (Olympus, Inc. Center Valley, PA), as described before ${ }^{44,46}$. Briefly, a $532-\mathrm{nm}$ laser beam (pulse repetition rate: $10 \mathrm{kHz}$ ) scanned the sample in an XY raster pattern using a pair of galvo mirrors $(6215 \mathrm{H}$, Cambridge Technologies, Lexington, MA). The laser beam was focused by using $10 \times(\mathrm{UPlan}$, Olympus Inc.) or 2.3 (Thorlabs, Newport, NJ) objectives from the bottom of the sample. The focal area of the transducers defined the field of view $-120 \mu \mathrm{m}$ for the $20-\mathrm{MHz}$ focused transducer $(\mathrm{V} 316,12 \mathrm{~mm}$ focal distance, Olympus-NDT Inc,) and $1.2 \mathrm{~mm}$ for the unfocused 3.5-MHz transducer (model 6528101, $4.5 \mathrm{~mm}$ diameter; Imasonic Inc., Besançon, France). DI water was placed in the dishes containing the spheroids to enable acoustic measurement. A computer with a high-speed digitizer (PCI-5124, 12-bit card, National Instruments, Austin, TX) was used to amplify and record the PA signals (5662B, Panametrics). A digital waveform generator (DG4062, Rigol, Beijing, China) enabled control over the mirrors and system synchronization. To perform wide-area imaging, a $1.2 \times$ objective and 3.5-MHz transducers were utilized in mosaic mode by shifting the position of the samples $(0.65 \mathrm{~mm}$ step) via a mechanical stage (Proscan II, Prior Scientific, Inc. Rockland, MA). Z-stack was used to determine the signal through the spheroids. The PA signal of the whole sphere was reconstituted using stacking and 3D stack images via NIH ImageJ.

Nanoparticle interaction on the sectioned sphere. After 5 days of formation and various incubation times with the nanoparticles, the spheroids were washed at least twice with 1x PBS and fixed by adding 4\% PFA for at least 1 hour at room temperature. The spheroids were then washed at least twice and embedded in cold molded agarose-derived ge ${ }^{56-58}$. The solid block was maintained under ethanol atmosphere until it was embedded in paraffin for microtomic slicing at a thickness of $\sim 10 \mu \mathrm{m}$. The PTM setup has been described in detail previously and is summarized here ${ }^{43,59}$. Briefly, PT microscopy was carried out on a custom-built platform based on an invert Olympus IX73 (Olympus America, Inc., Central Valley, PA) using a 3-wavelength RGB Combiner (RGB46HF, Thorlabs, Newton, NJ) to combine 488-nm (fluorescence excitation: IQ1C45 (488-60) laser diode, Power Technology, Little Rock, AR), 532-nm (PT pump: LabSpec 532 nm DPSS Laser. Laserglow Technologies, LLS series, Toronto, Canada) and 635-nm (PT probe: LP637 SM Fiber-Pigtailed Laser Diode, Thorlabs, Newton, NJ) laser beams into a single mode fiber (Fig. S5). High-resolution confocal fluorescence and PTM imaging were performed at the same time by guiding laser beams using galvo-mirrors (GVSM002, Thorlabs, Newton, NJ) across the sample. Probe beam intensity was collected via a 40x objective placed above the sample and measured by an amplified photodiode (PDA10A, Thorlabs, Newton, NJ). The PT signal was extracted from the probe beam intensity modulation using a digital lock-in amplifier (MFLI, $500 \mathrm{kHz}, 60 \mathrm{MSa} / \mathrm{s}$, Zurich Instruments, Switzerland) and recorded by LabView software. Conventional fluorescent imaging was done with a CCD DP80 camera (Olympus America, Central Valley, PA). The sliced spheroids were measured slice-by-slice. The PTM images were taken from a $47 \times 47 \mu \mathrm{m}$ area. Each spheroid required several PTM images, which were then reconstructed into one image by stitching in Adobe Illustrator CC and overlapped with the fluorescence signal. Final image brightness/ contrast was adjusted using Adobe Photoshop CC. The plot line and 3D profile were analyzed using ImageJ.

Received: 17 October 2019; Accepted: 24 January 2020;

Published online: 25 February 2020

\section{References}

1. Brigger, I., Dubernet, C. \& Couvreur, P. Nanoparticles in cancer therapy and diagnosis. Advanced drug delivery reviews 64, 24-36, https://doi.org/10.1016/j.addr.2012.09.006 (2012).

2. Orza, A., Casciano, D. \& Biris, A. Nanomaterials for targeted drug delivery to cancer stem cells. Drug metabolism reviews 46, 191-206 (2014)

3. Bhaw-Luximon, A. \& Jhurry, D. New avenues for improving pancreatic ductal adenocarcinoma (PDAC) treatment: Selective stroma depletion combined with nano drug delivery. Cancer letters 369, 266-273 (2015).

4. Dimou, A., Syrigos, K. N. \& Saif, M. W. Overcoming the stromal barrier: technologies to optimize drug delivery in pancreatic cancer. Therapeutic Advances in Medical Oncology 4, 271-279 (2012).

5. Bahrami, A. et al. Targeted Stroma in Pancreatic Cancer: Promises and Failures of Target Therapies. Journal of cellular physiology (2017).

6. Gao, S. et al. Engineering Nanoparticles for Targeted Remodeling of the Tumor Microenvironment to Improve Cancer Immunotherapy. Theranostics 9, 126 (2019).

7. Surendran, S. P., Moon, M. J., Park, R. \& Jeong, Y. Y. Bioactive nanoparticles for cancer immunotherapy. International journal of molecular sciences 19, 3877 (2018).

8. Han, X. et al. Reversal of pancreatic desmoplasia by re-educating stellate cells with a tumour microenvironment-activated nanosystem. Nature communications $\mathbf{9}, 3390$ (2018).

9. Saha, S. et al. Gold Nanoparticle Reprograms Pancreatic Tumor Microenvironment and Inhibits Tumor Growth. ACS nano (2016).

10. Huang, B.-W. \& Gao, J.-Q. Application of 3D cultured multicellular spheroid tumor models in tumor-targeted drug delivery system research. Journal of Controlled Release 270, 248-259 (2017).

11. Senthebane, D. A. et al. The Role of Tumor Microenvironment in Chemoresistance: To Survive, Keep Your Enemies Closer. International journal of molecular sciences 18, 1586 (2017). 
12. Au, M., Emeto, T. I., Power, J., Vangaveti, V. N. \& Lai, H. C. Emerging Therapeutic Potential of Nanoparticles in Pancreatic Cancer: A Systematic Review of Clinical Trials. Biomedicines 4, 20 (2016).

13. Öhlund, D. et al. Distinct populations of inflammatory fibroblasts and myofibroblasts in pancreatic cancer. The Journal of experimental medicine 214, 579-596 (2017).

14. Bolm, L. et al. The Role of Fibroblasts in Pancreatic Cancer: Extracellular Matrix Versus Paracrine Factors. Journal of translational oncology 10, 578-588 (2017).

15. Guo, S. \& Deng, C.-X. Effect of Stromal Cells in Tumor Microenvironment on Metastasis Initiation. International Journal of Biological Sciences 14, 2083-2093 (2018).

16. Apte, M. V., Wilson, J. S., Lugea, A. \& Pandol, S. J. A starring role for stellate cells in the pancreatic cancer microenvironment. Gastroenterology 144, 1210-1219 (2013).

17. Sada, M. et al. Hypoxic stellate cells of pancreatic cancer stroma regulate extracellular matrix fiber organization and cancer cell motility. Cancer letters 372, 210-218 (2016).

18. Kadel, D. et al. Current perspectives of cancer-associated fibroblast in therapeutic resistance: potential mechanism and future strategy, 1-15 (2019).

19. Bynigeri, R. R. et al. Pancreatic stellate cell: Pandora’s box for pancreatic disease biology. World journal of gastroenterology 23, 382-405 (2017)

20. Habisch, H., Zhou, S., Siech, M. \& Bachem, M. G. Interaction of stellate cells with pancreatic carcinoma cells. cancers 2, 1661-1682 (2010).

21. Sherman, M. H. Stellate Cells in Tissue Repair, Inflammation, and Cancer. J Annual review of cell developmental biology (2018).

22. Ware, M. J. et al. Generation of an in vitro 3D PDAC stroma rich spheroid model. Biomaterials 108, 129-142 (2016).

23. Rubiano, A. et al. Viscoelastic properties of human pancreatic tumors and in vitro constructs to mimic mechanical properties. Acta biomaterialia (2017).

24. Karnevi, E., Rosendahl, A. H., Hilmersson, K. S., Saleem, M. A. \& Andersson, R. Impact by pancreatic stellate cells on epithelialmesenchymal transition and pancreatic cancer cell invasion: Adding a third dimension in vitro. Experimental Cell Research 346, 206-215 (2016).

25. Priwitaningrum, D. L. et al. Tumor stroma-containing 3D spheroid arrays: A tool to study nanoparticle penetration. Journal of Controlled Release (2016)

26. Cox, M. C., Reese, L. M., Bickford, L. R. \& Verbridge, S. S. Toward the broad adoption of 3D tumor models in the cancer drug pipeline. ACS Biomaterials Science \& Engineering 1, 877-894 (2015).

27. Darrigues, E. et al. Raman spectroscopy using plasmonic and carbon-based nanoparticles for cancer detection, diagnosis, and treatment guidance.Part 1: Diagnosis. Drug Metabolism Reviews 49, 212-252, https://doi.org/10.1080/03602532.2017.1302465 (2017).

28. Darrigues, E. et al. Raman spectroscopy using plasmonic and carbon-based nanoparticles for cancer detection, diagnosis, and treatment guidance. Part 2: Treatment. Drug Metabolism Reviews 49, 253-283, https://doi.org/10.1080/03602532.2017.1307387 (2017).

29. Nima, Z. A. et al. Applications of surface-enhanced Raman scattering in advanced bio-medical technologies and diagnostics. Drug metabolism reviews $\mathbf{4 6}, 155-175$ (2014).

30. Phan, T. T. V. et al. Photoacoustic Imaging-Guided Photothermal Therapy with Tumor-Targeting HA-FeOOH@ PPy Nanorods. Scientific reports 8, 8809 (2018).

31. Wu, C. Y. et al. MR imaging of human pancreatic cancer xenograft labeled with superparamagnetic iron oxide in nude mice. Contrast media \& molecular imaging 7, 51-58 (2012).

32. Durr, N. J. et al. Two-photon luminescence imaging of cancer cells using molecularly targeted gold nanorods. Nano letters 7 , 941-945 (2007)

33. Nima, Z. A. et al. Quantification of cellular associated graphene and induced surface receptor responses. Nanoscale 11, 932-944 (2019).

34. Darrigues, E. et al. 3D cultures for modeling nanomaterial-based photothermal therapy. Nanoscale Horizons, https://doi. org/10.1039/C9NH00628A (2020)

35. Rane, T. D. \& Armani, A. M. Two-Photon Microscopy Analysis of Gold Nanoparticle Uptake in 3D Cell Spheroids. Plos one 11, e0167548 (2016)

36. Huang, Y., He, S., Cao, W., Cai, K. \& Liang, X.-J. Biomedical nanomaterials for imaging-guided cancer therapy. Nanoscale 4, 6135-6149 (2012).

37. Lazzari, G. et al. Light sheet fluorescence microscopy versus confocal microscopy: in quest of a suitable tool to assess drug and nanomedicine penetration into multicellular tumor spheroids. European Journal of Pharmaceutics and Biopharmaceutics 142, 195-203, https://doi.org/10.1016/j.ejpb.2019.06.019 (2019).

38. Brancato, V. et al. 3D tumor microtissues as an in vitro testing platform for microenvironmentally-triggered drug delivery systems. Acta Biomaterialia 57, 47-58 (2017)

39. Koltai, T. Cancer: fundamentals behind pH targeting and the double-edged approach. OncoTargets and therapy 9, 6343 (2016).

40. Wang, M. et al. Magnetically and $\mathrm{pH}$ dual responsive dendrosomes for tumor accumulation enhanced folate-targeted hybrid drug delivery. Journal of Controlled Release 232, 161-174 (2016).

41. Wu, W. et al. Endogenous $\mathrm{pH}$-responsive nanoparticles with programmable size changes for targeted tumor therapy and imaging applications. Theranostics 8, 3038-3058 (2018).

42. Hermanson, G. T. In Bioconjugate Techniques (Third Edition) (ed. Greg T. Hermanson) 395-463 (Academic Press, 2013).

43. Nedosekin, D. A., Galanzha, E. I., Ayyadevara, S., Shmookler Reis, R. J. \& Zharov, V. P. Photothermal confocal spectromicroscopy of multiple cellular chromophores and fluorophores. Biophysical journal 102, 672-681 (2012).

44. Shao, J. et al. Photothermal nanodrugs: potential of TNF-gold nanospheres for cancer theranostics. Scientific reports 3, 1293-1293 (2013).

45. Nima, Z. A. et al. Circulating tumor cell identification by functionalized silver-gold nanorods with multicolor, super-enhanced SERS and photothermal resonances. Scientific Reports 4, 4752 (2014).

46. Jenkins, S. V. et al. Triple-negative breast cancer targeting and killing by EpCAM-directed, plasmonically active nanodrug systems. NPJ precision oncology $1,27-27$ (2017).

47. Nima, Z. A. et al. Targeting nano drug delivery to cancer cells using tunable, multi-layer, silver-decorated gold nanorods. Journal of applied toxicology 37, 1370-1378 (2017).

48. Vang, K. B. et al. Modifying Dendritic Cell Activation with Plasmonic Nano Vectors. Scientific Reports 7, 5513 (2017)

49. Nikoobakht, B. \& El-Sayed, M. A. Preparation and Growth Mechanism of Gold Nanorods (NRs) Using Seed-Mediated Growth Method. Chemistry of Materials 15, 1957-1962 (2003).

50. Gong, C., Shan, M., Li, B. \& Wu, G. A pH and redox dual stimuli-responsive poly (amino acid) derivative for controlled drug release. Colloids and Surfaces B: Biointerfaces 146, 396-405 (2016).

51. Li, Q.-L. et al. $\mathrm{pH}$ and glutathione dual-responsive dynamic cross-linked supramolecular network on mesoporous silica nanoparticles for controlled anticancer drug release. ACS applied materials \& interfaces 7, 28656-28664 (2015).

52. Durymanov, M., Kroll, C., Permyakova, A. \& Reineke, J. Role of Endocytosis in Nanoparticle Penetration of 3D Pancreatic Cancer Spheroids. Molecular Pharmaceutics 16, 1074-1082 (2019). 
53. Nedosekin, D. A. et al. Photoacoustic and photothermal detection of circulating tumor cells, bacteria and nanoparticles in cerebrospinal fluid in vivo and ex vivo. Journal of biophotonics 6, 523-533 (2013).

54. Nedosekin, D. A., Shashkov, E. V., Galanzha, E. I., Hennings, L. \& Zharov, V. P. Photothermal multispectral image cytometry for quantitative histology of nanoparticles and micrometastasis in intact, stained and selectively burned tissues. Cytometry Part A 77, 1049-1058 (2010).

55. Kota, J., Hancock, J., Kwon, J. \& Korc, M. Pancreatic cancer: Stroma and its current and emerging targeted therapies. Cancer letters (2017).

56. De Hoogt, R. et al. Protocols and characterization data for 2D, 3D, and slice-based tumor models from the PREDECT project. Scientific data 4, 170170 (2017).

57. Wiley, L. A., Beebe, D. C., Mullins, R. F., Stone, E. M. \& Tucker, B. A. A Method for Sectioning and Immunohistochemical Analysis of Stem Cell-Derived 3-D Organoids. Current protocols in stem cell biology 37, 1C. 19.11-11C. 19.11 (2016).

58. Wong, C., Vosburgh, E., Levine, A. J., Cong, L. \& Xu, E. Y. Human neuroendocrine tumor cell lines as a three-dimensional model for the study of human neuroendocrine tumor therapy. JoVE (Journal of Visualized Experiments), e4218 (2012).

59. Zharov, V. P. Ultrasharp nonlinear photothermal and photoacoustic resonances and holes beyond the spectral limit. Nature Photonics 5, 110 (2011).

\section{Acknowledgements}

We acknowledge support from the Center for Advanced Surface Engineering, under the National Science Foundation Grant No. IIA-1457888 and the Arkansas EPSCoR Program, ASSET III. We are very grateful to Emily Davis for the review and assistance with the technical editing of this manuscript. We want to thank Dr. Analiz Rodriguez, from the University of Arkansas for Medical Sciences, Winthrop P. Rockefeller Cancer Institute, Department of Neurosurgery, for allowing us access to her lab and to the BioTek Cytation 5 instrument. We are grateful to the Experimental Pathology Core at the University of Arkansas for Medical Sciences for their assistance in slicing the spheroids.

\section{Author contributions}

E.D. performed the synthesis and functionalization of the nanoparticles, evaluated the drug-release. Z.A.N. assisted E.D. with the AuNRs synthesis and with the AFM images. E.D. realized the cell culture, formation and maintenance of spheroids and processed to the incubation of the AuNRs with the spheroids. E.D. performed the imaging of the spheroids with the Cytation 5 and the preparation of spheroids for slicing. D.A.N. performed the PAM and high-resolution mode PTM imaging. F.W. and K.M.A. helped with TEM sample preparation and imaging; performed the AFM; and E.D., Z.A.N., D.A.N., F.W., K.M.A., V.P.Z. and A.S.B. wrote the manuscript and contributed to the ideas presented within. All the authors reviewed the manuscript.

\section{Competing interests}

The authors declare no competing interests.

\section{Additional information}

Supplementary information is available for this paper at https://doi.org/10.1038/s41598-020-59226-6.

Correspondence and requests for materials should be addressed to E.D. or A.S.B.

Reprints and permissions information is available at www.nature.com/reprints.

Publisher's note Springer Nature remains neutral with regard to jurisdictional claims in published maps and institutional affiliations.

(c) (i) Open Access This article is licensed under a Creative Commons Attribution 4.0 International License, which permits use, sharing, adaptation, distribution and reproduction in any medium or format, as long as you give appropriate credit to the original author(s) and the source, provide a link to the Creative Commons license, and indicate if changes were made. The images or other third party material in this article are included in the article's Creative Commons license, unless indicated otherwise in a credit line to the material. If material is not included in the article's Creative Commons license and your intended use is not permitted by statutory regulation or exceeds the permitted use, you will need to obtain permission directly from the copyright holder. To view a copy of this license, visit http://creativecommons.org/licenses/by/4.0/.

(C) The Author(s) 2020 